\title{
Neutron and electron electric dipole moments (EDMs)
}

\author{
Mike Tarbutt
}

Centre for Cold Matter - Imperial College London - United Kingdom 


\title{
Imperial College
}

London

\section{Neutron and electron electric dipole moments (EDMs)}

\author{
Mike Tarbutt \\ Centre for Cold Matter \\ Imperial College London
}

Lectures given at QED2012, Cargèse, Corsica, April 18 ${ }^{\text {th }}-19^{\text {th }} 2012$ 


\section{What is an EDM?}

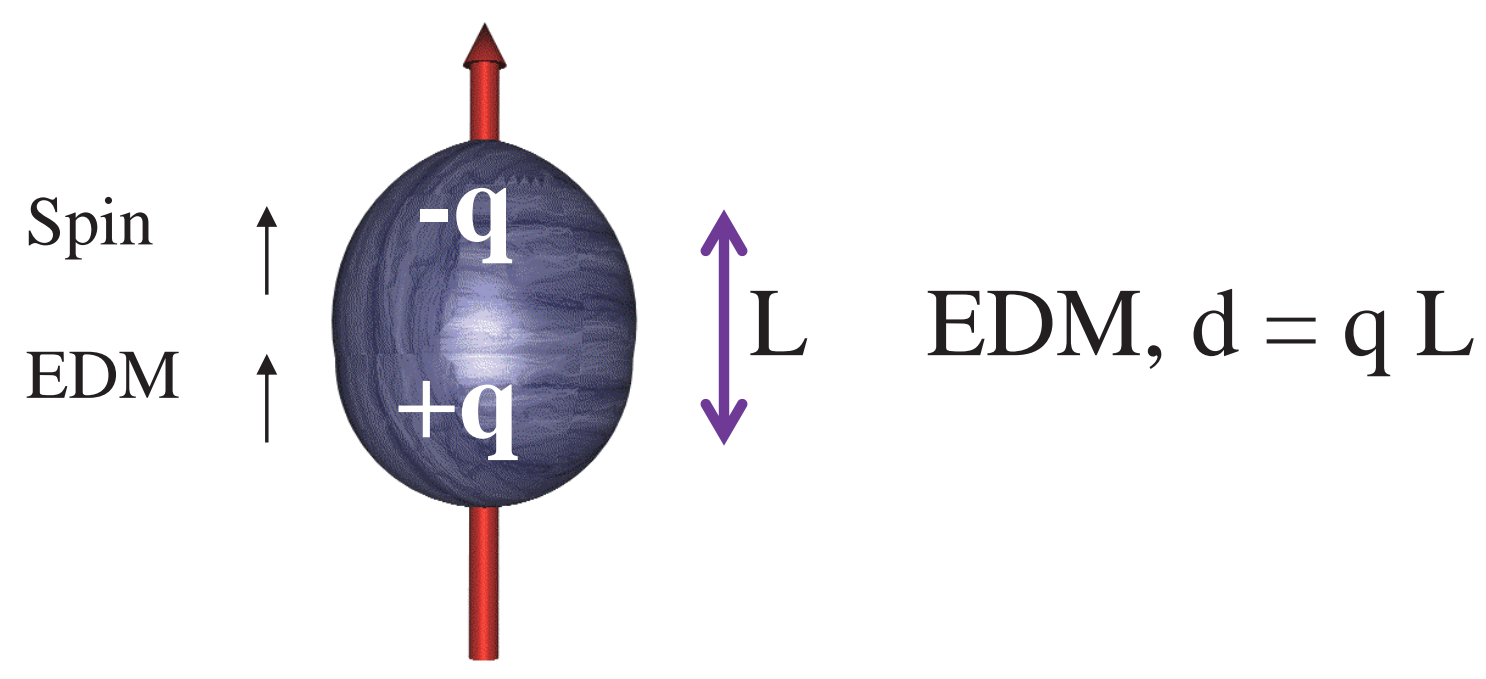

A magnetic dipole moment $\mu$ in a magnetic field $\mathbf{B}$ has energy $-\mu . \mathbf{B}$

An electric dipole moment $\mathbf{d}$ in an electric field $\mathbf{E}$ has energy - $\mathbf{d} . \mathbf{E}$

N.B. Both $\mu$ and $\mathbf{d}$ are parallel (or antiparallel) to the spin 


\section{What is an EDM?}

\section{Neutron}

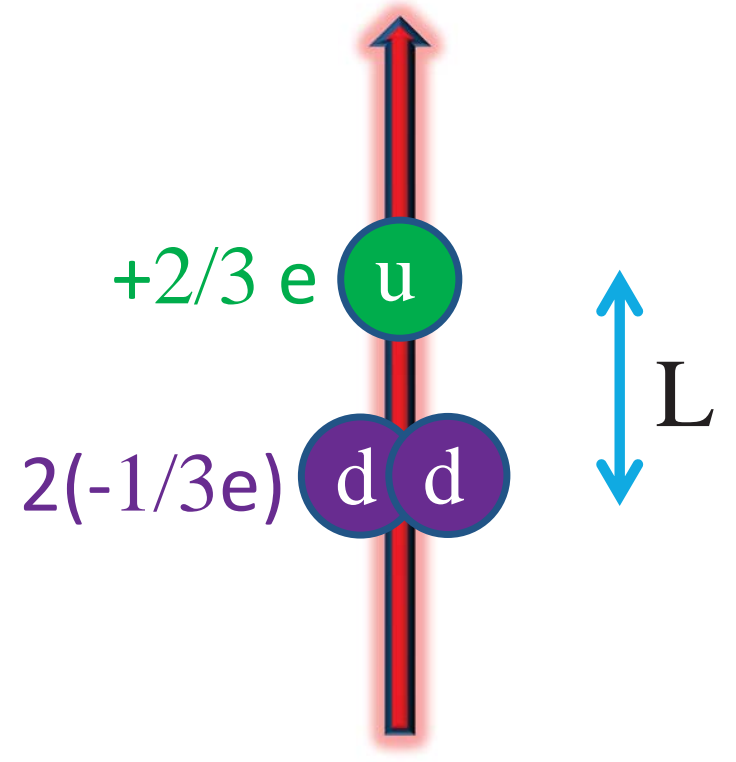

Example: If $L \sim 0.1$ times neutron radius: $d_{n} \sim 10^{-15}$ e.cm

Experimental upper limit: $\left|d_{n}\right|<2.9 \times 10^{-26}$ e.cm

\section{Electron}




\title{
Setting the scale
}

\author{
Suppose the neutron EDM is $d_{n}=2.0 \times 10^{-26} \mathrm{e} . \mathrm{cm}$ \\ (just below current upper limit)
}

In an electric field of $10 \mathrm{kV} / \mathrm{cm}$ the interaction energy is:

\subsection{2 atto-eV or $0.05 \mu \mathrm{Hz}$ or $0.002 \mathrm{f} \mathrm{cm}^{-1}$ or $0.002 \mathrm{fK}$.}

The neutron has a magnetic dipole moment. The magnetic field that produces the same interaction energy is only $\mathbf{2} \mathrm{fT}$.

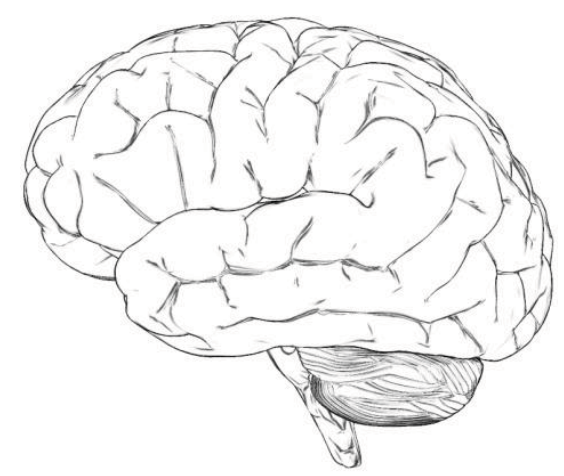


A non-zero EDM violates time-reversal symmetry

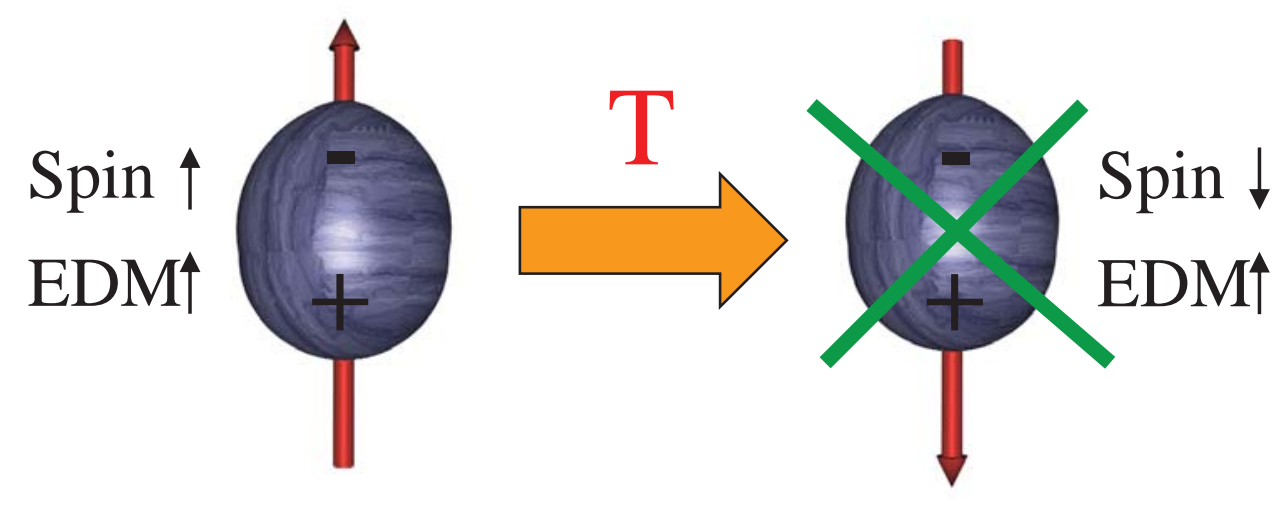

Either $\mathrm{d}_{\mathrm{e}}=0$, or $X$ 


\section{Discrete symmetries}

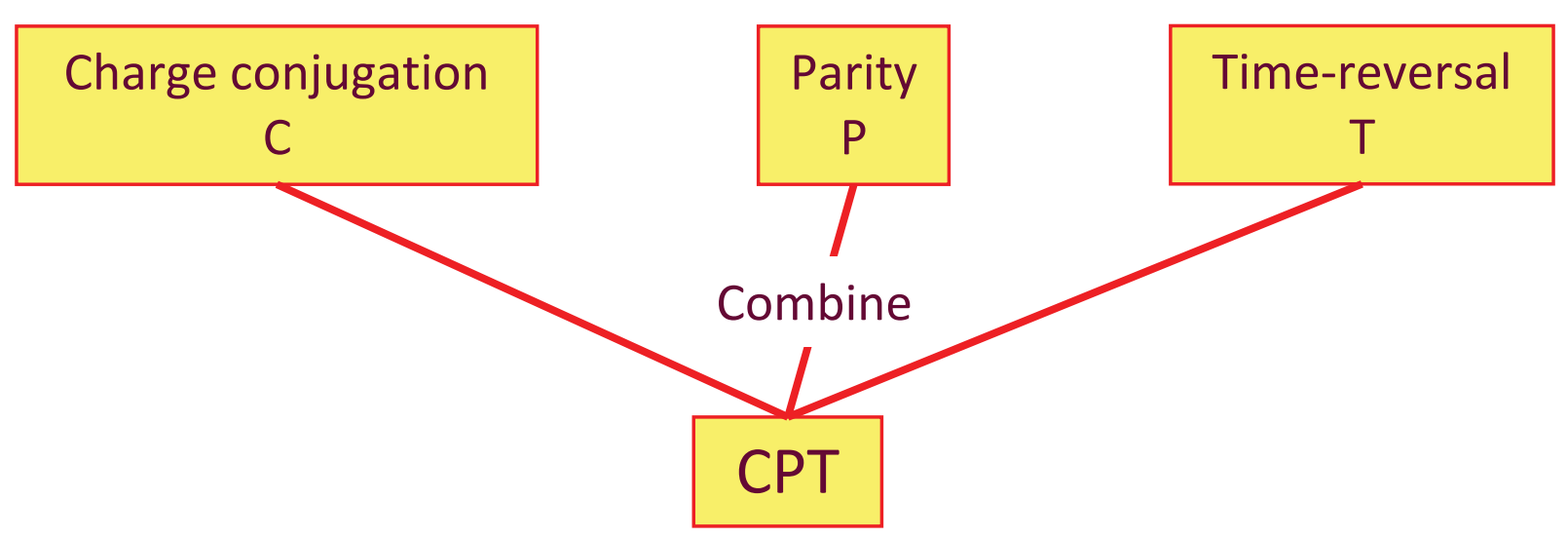

All local, Lorentz-invariant quantum field theories are invariant under CPT

\begin{tabular}{|c|c|c|}
\hline CD vislation & CPT theorem & T violation \\
\hline
\end{tabular}

A non-zero EDM violates CP symmetry 


\section{We need CP violation!}

The entire observable universe appears to be made of matter

$\mathrm{CP}$ violation is necessary to explain how the universe came to be this way

$\mathrm{CP}$ violation in the Standard Model is insufficient to explain the observed matter-antimatter asymmetry

Theories beyond the Standard Model introduce new CP-violating interactions

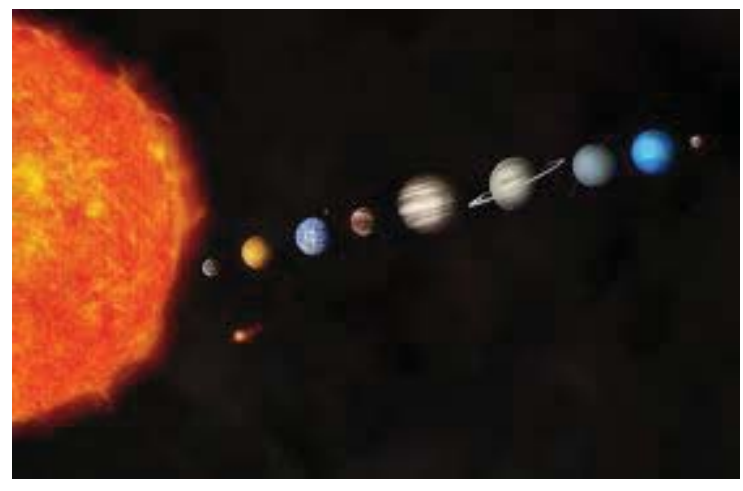

Our solar system -2 billion billion billion tonnes of matter

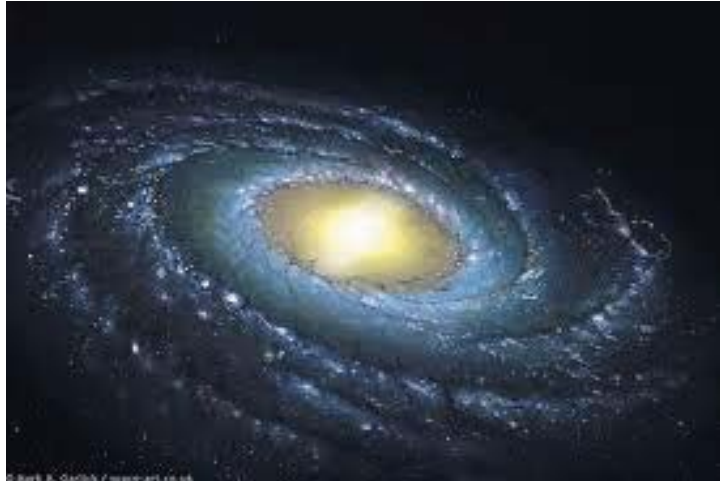

Our galaxy - 200 billion stars

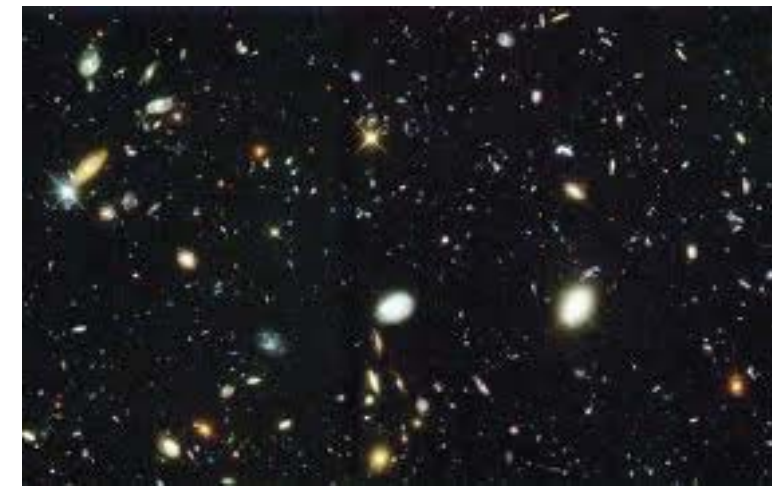

Observable universe -80 billion galaxies 


\section{EDMs, the Standard Model and beyond}

$-d(\mu) \leq 7 \times 10^{-19}$

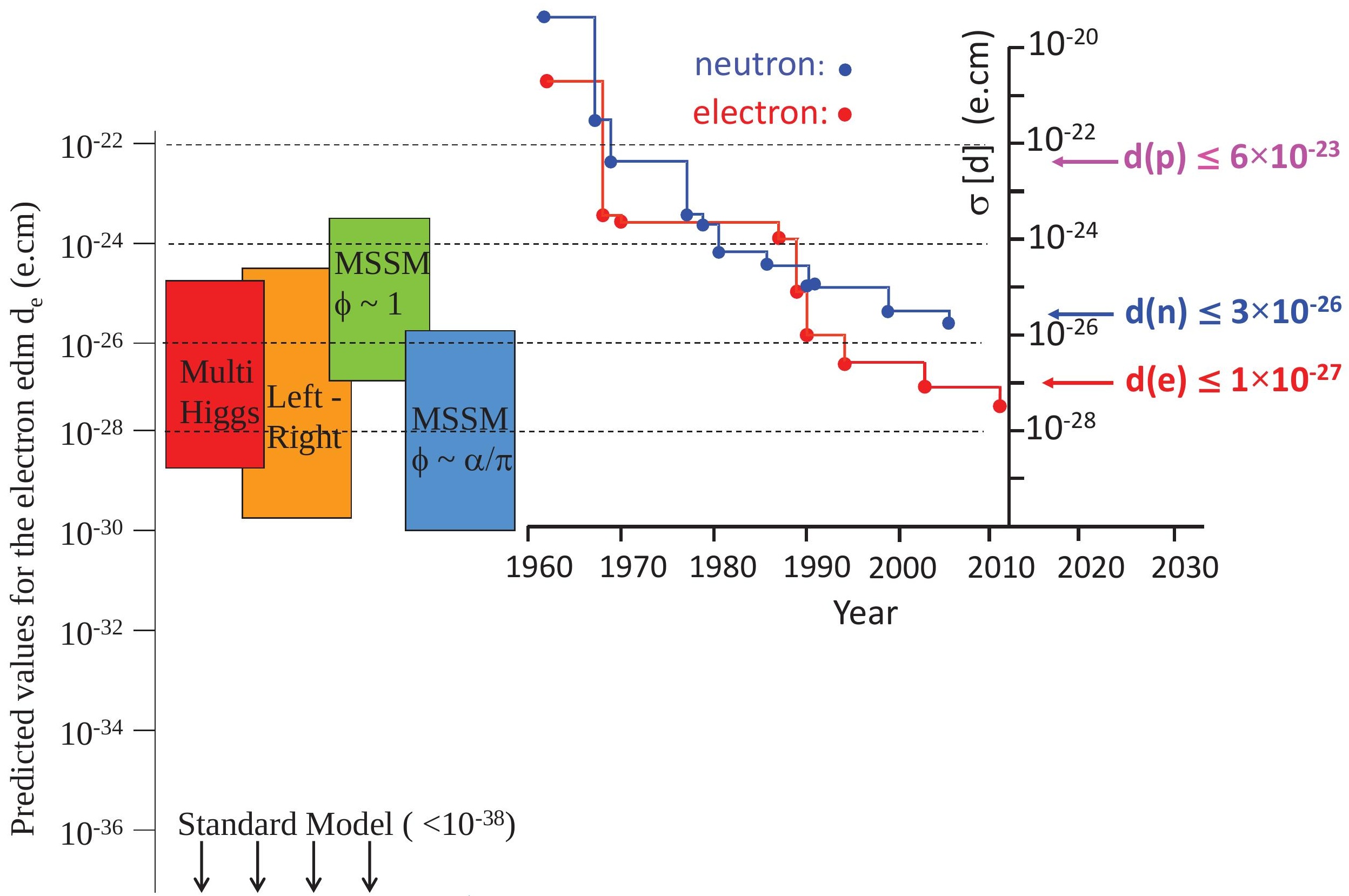




\section{Summary of motivations for measuring EDMs}

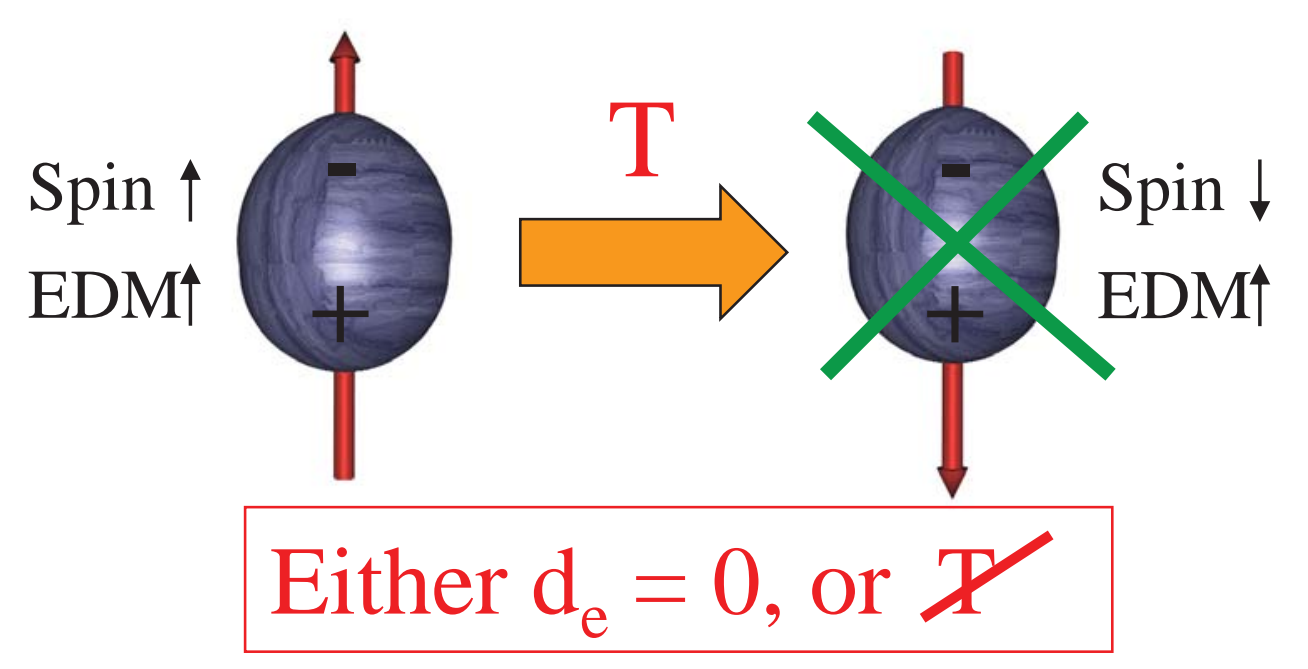

A non-zero EDM breaks the symmetry between matter and antimatter (CP symmetry)
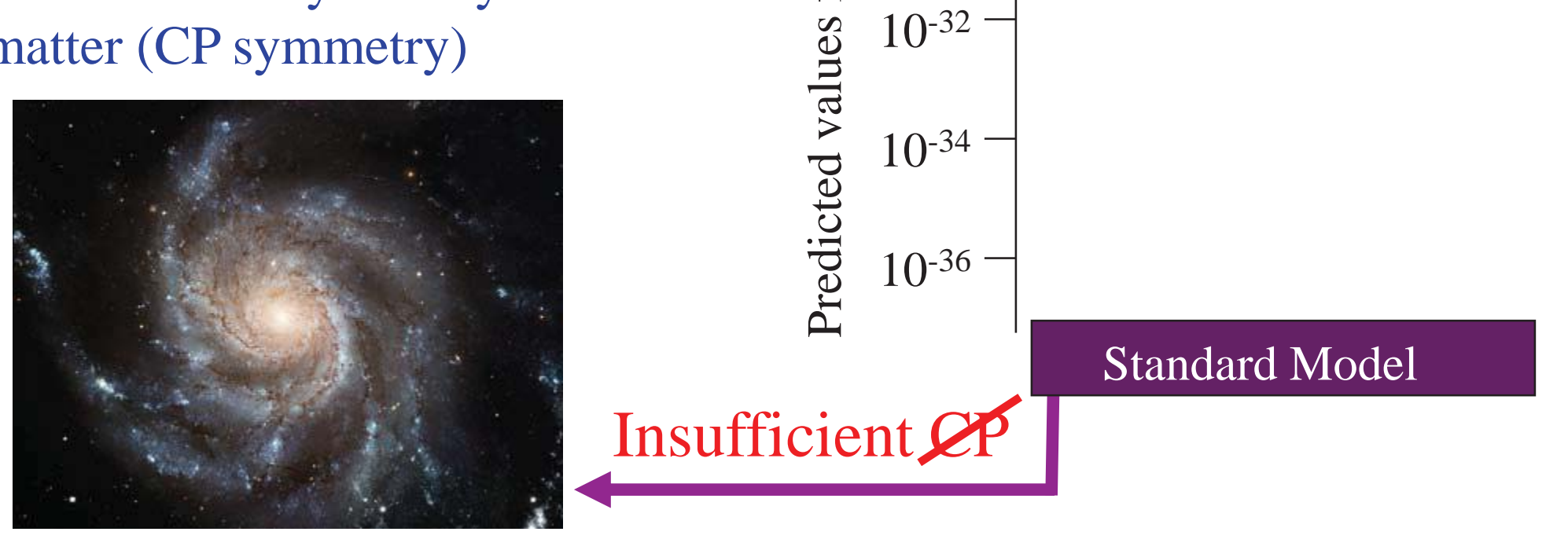


\section{How to measure EDMs - spin precession}

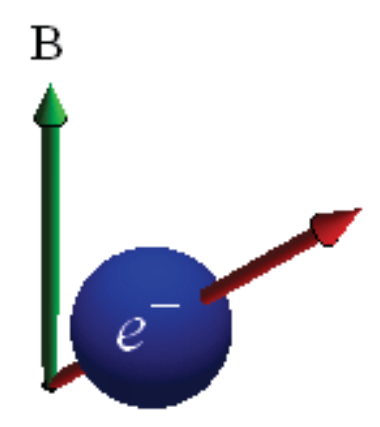

Particle precessing in a magnetic field

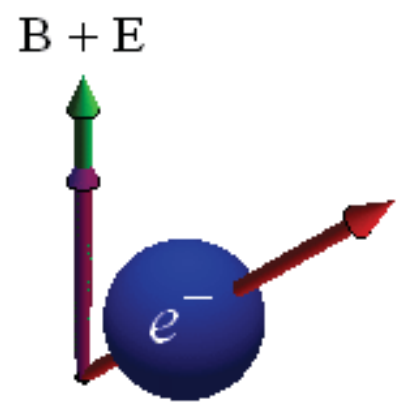

Particle precessing in parallel magnetic and electric fields precession rate increases due to additional EDM interaction

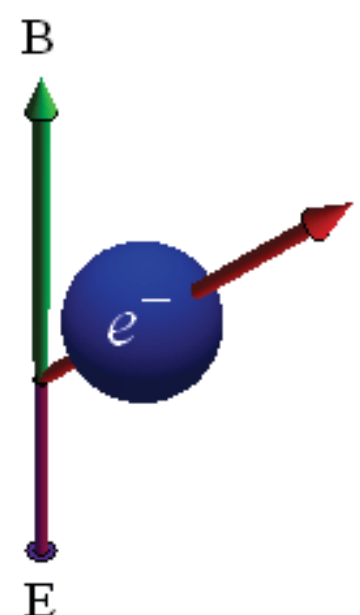

Particle precessing in anti-parallel magnetic and electric fields precession rate decreases due to additional EDM interaction

Measure change in frequency when electric field direction is reversed this determines the EDM 


\section{How to measure EDMs}

$$
H=-\mu \cdot B-d \cdot E
$$
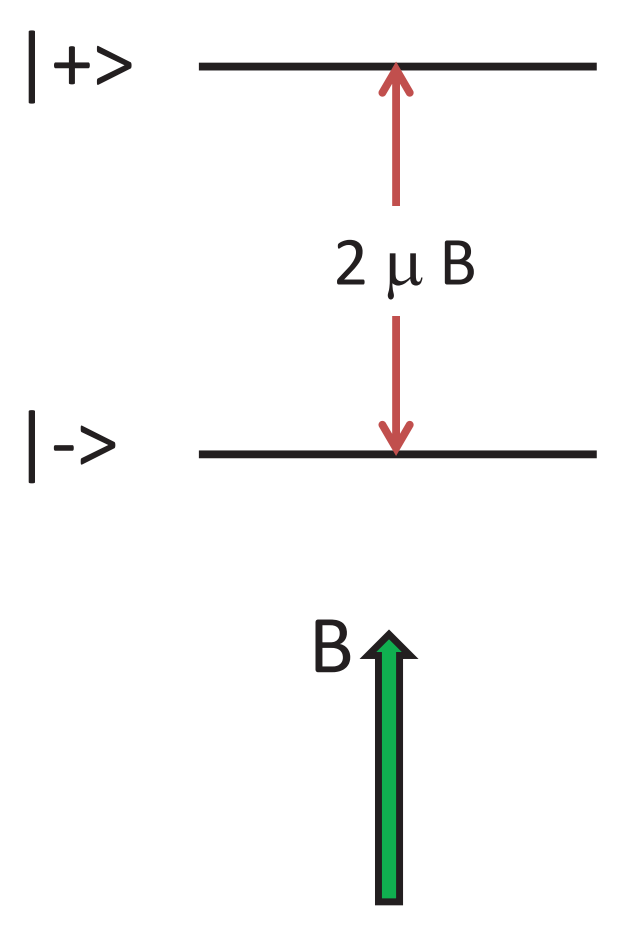
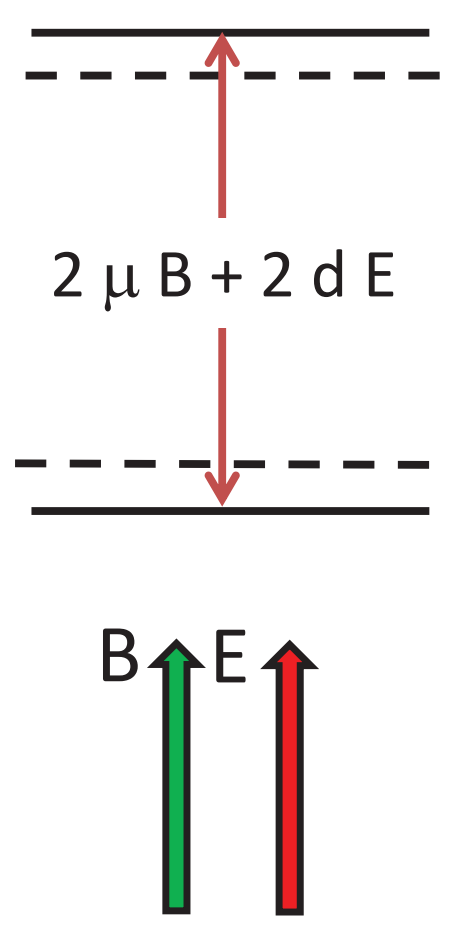

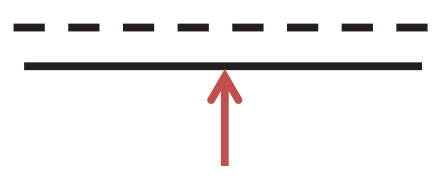

$2 \mu B-2 d E$
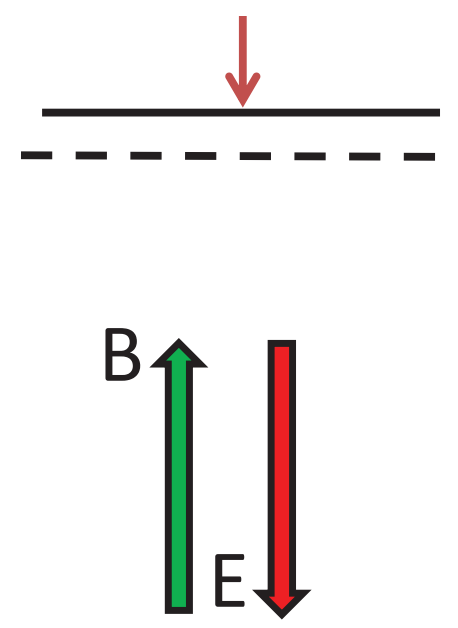

Measure change in frequency when electric field direction is reversed this is $4 \mathrm{~d} \mathrm{E}$ - determines the EDM, $\mathrm{d}$ 


\section{Measuring the neutron EDM using a neutron beam}

1.

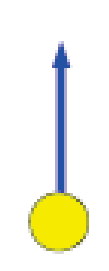

"Spin up" neutron...

2.

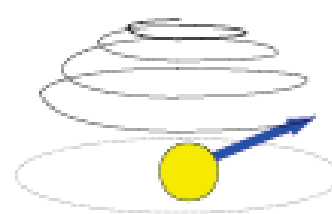

3.

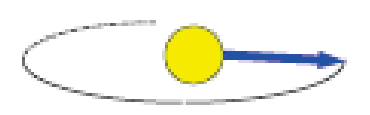

4.

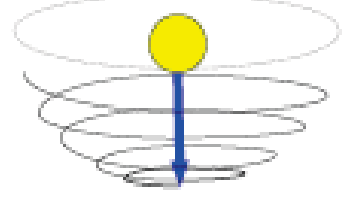

Second $90^{\circ}$ spin-flip pulse spin-polarized neutron beam

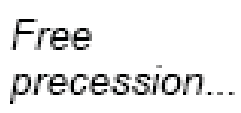

\section{$90^{\circ}$ spin-flip} pulse applied.. precession...

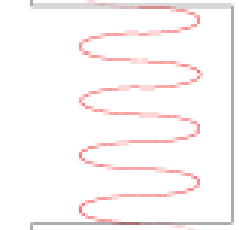

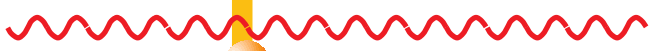
$2^{\text {nd }}$ rf pulse

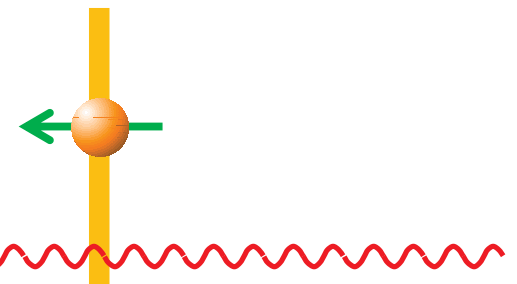

$1^{\text {st }}$ rf pulse

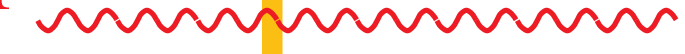

\section{B} $\mathbf{h f}_{ \pm}=2 \mu \mathrm{B} \pm \mathbf{2} \mathbf{d ~ E}$

\section{analyze spin direction}




\section{First EDM measurement}

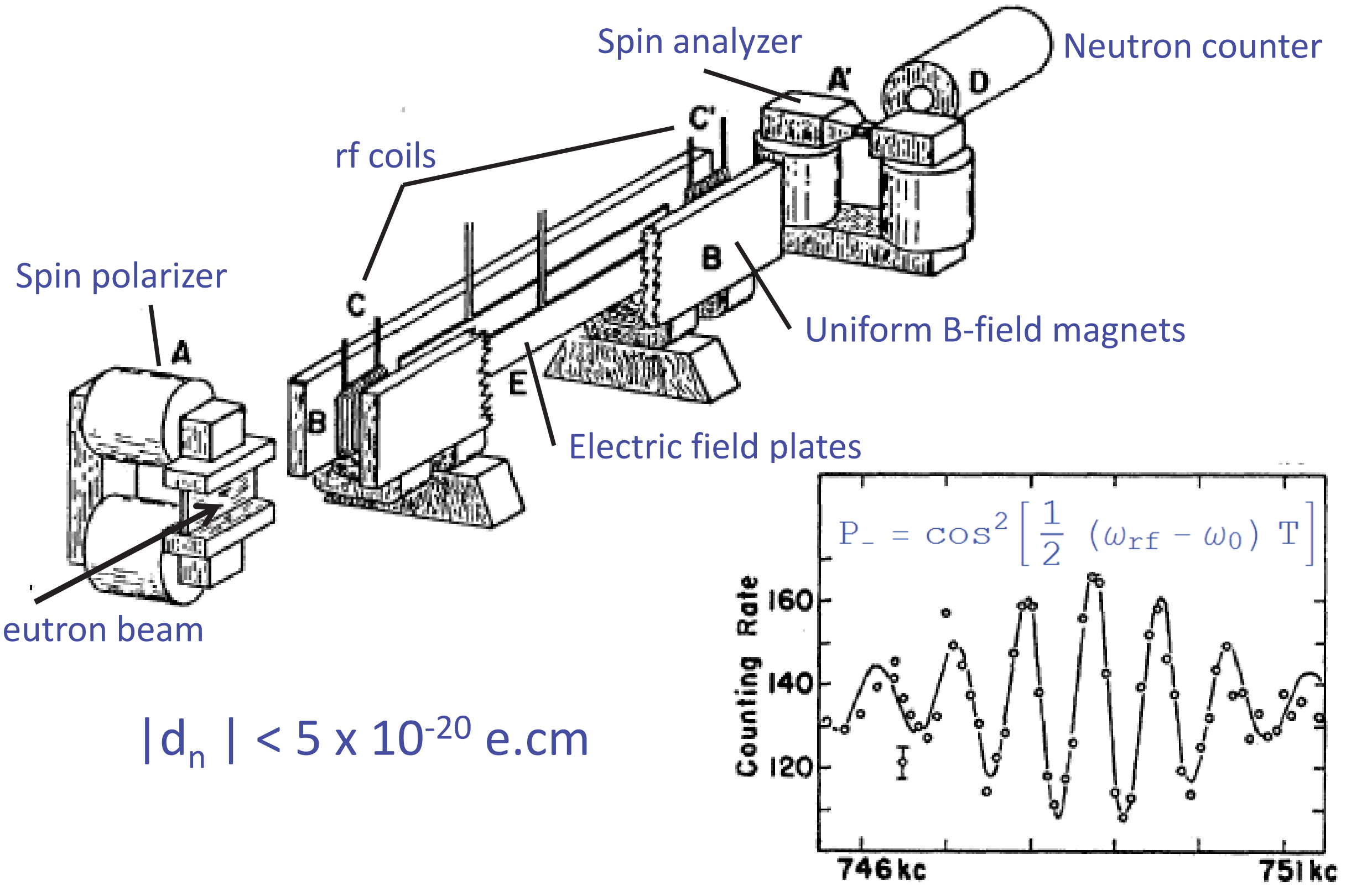

J.H. Smith, E.M. Purcell and N.F. Ramsey, Phys. Rev. 108, 120 (1957) 


\section{How to make better measurements}

Measure the period of a pendulum by three different methods:

(1) Measure time for 1 swing

(2) Measure time for 1 swing; repeat 100 times

(3) Measure time for 100 swings

Method (2) is 10 times more precise than method (1)

Method (3) is 100 times more precise than method (1)

Let it precess for longer!

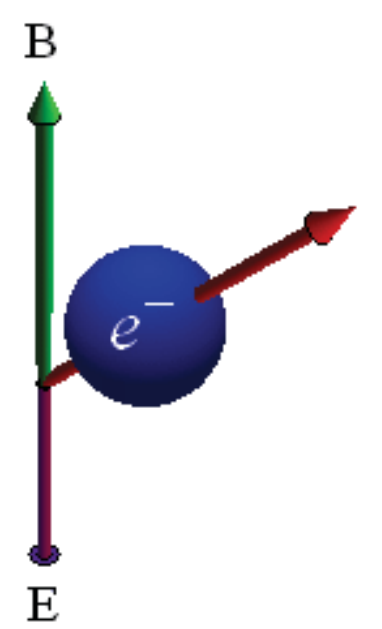




\section{How to make a sensitive EDM measurement}

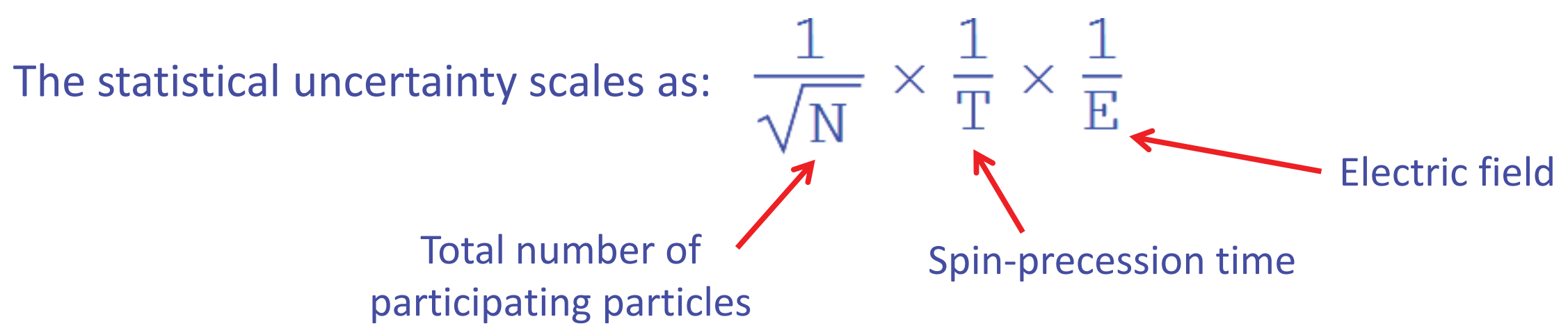

(1) Make $\mathrm{N}$ as big as possible (but note square-root scaling)

(2) Make T as big as possible

(3) Make E as big as possible (but there are limits!)

But note well - you will only reach the statistical noise limit if you also:

(4) Eliminate excess noise (particularly magnetic noise) - more on this later

(5) Eliminate systematic effects that mimic the EDM - more on this later 


\section{Problems with beam experiments}

(1) $T$ is limited by the length and speed to a few ms

(2) Huge systematic effect due to motional magnetic field

Motional magnetic field: $\boldsymbol{B}_{\text {mot }}=-\frac{1}{c^{2}}(\boldsymbol{v} \times \boldsymbol{E})$

$\boldsymbol{-} \boldsymbol{\mu} . \boldsymbol{B}_{\text {mot }}$ is an energy shift that changes sign when $\mathrm{E}$ is reversed

If the applied $E$ and $B$ fields are not perfectly parallel (and they never are), this appears as a false EDM. 


\section{From beams to bottles}

Slow neutrons bounce off walls!

There's a material-specific critical velocity, $\mathrm{v}_{\mathrm{c}}$ (e.g. $\sim 7 \mathrm{~m} / \mathrm{s}$ for $\mathrm{Ni}, \mathrm{Be}, \mathrm{BeO}$ )

Neutrons with $v<v_{c}$ are called ultracold neutrons (UCN) - they reflect elastically under any angle of incidence

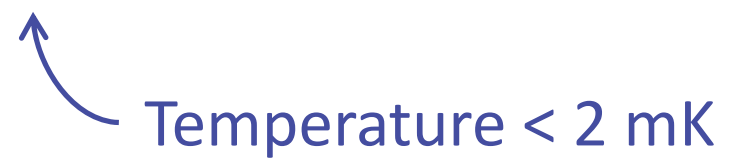

Using bottled neutrons the spin precession time T can be very long, ultimately limited by the neutron lifetime (about 15 minutes).

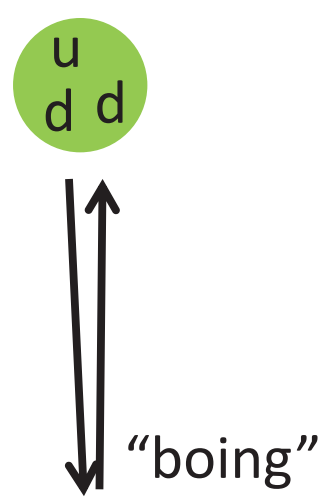




\section{Generating ultracold neutrons - neutron turbine}

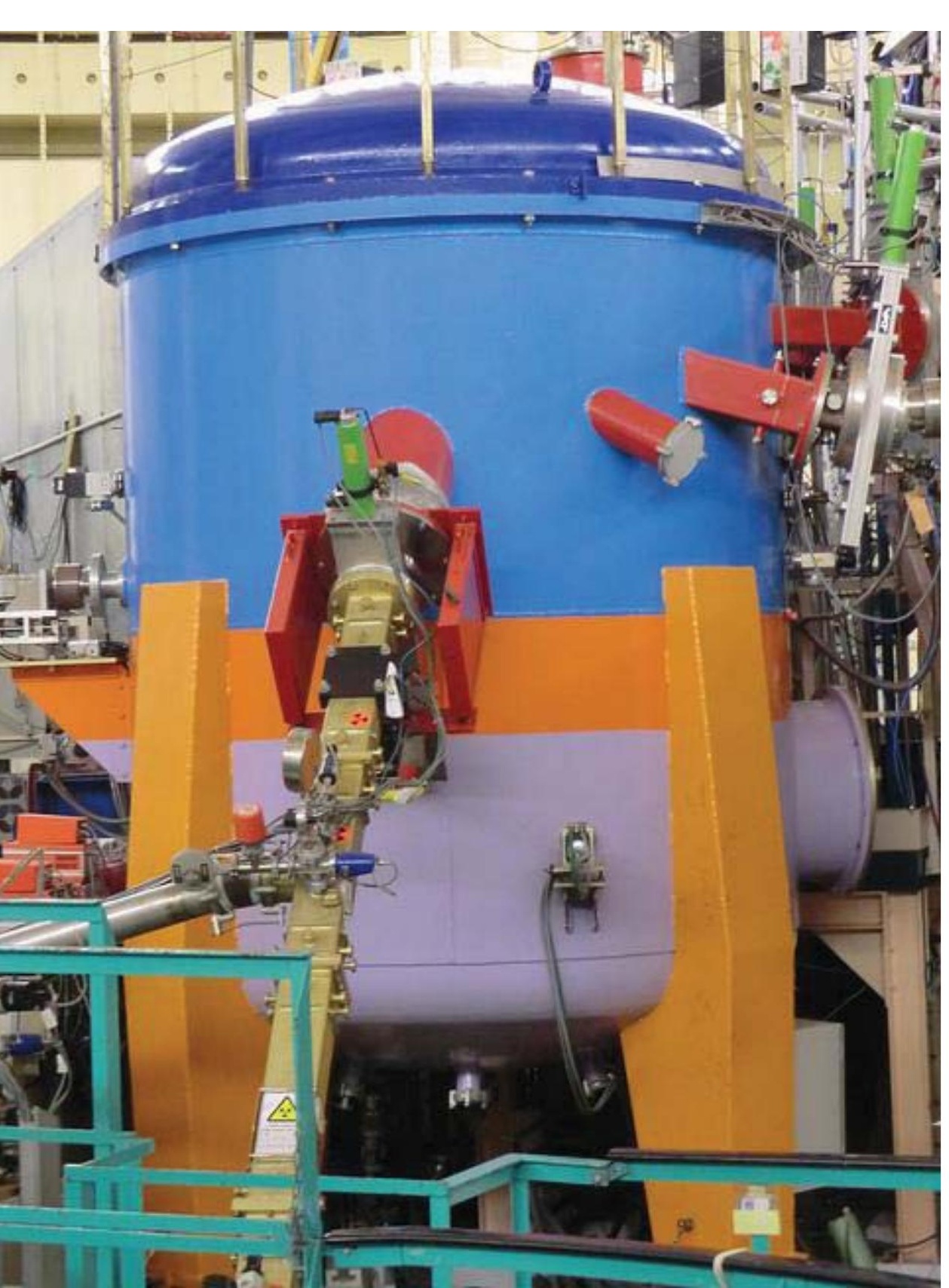

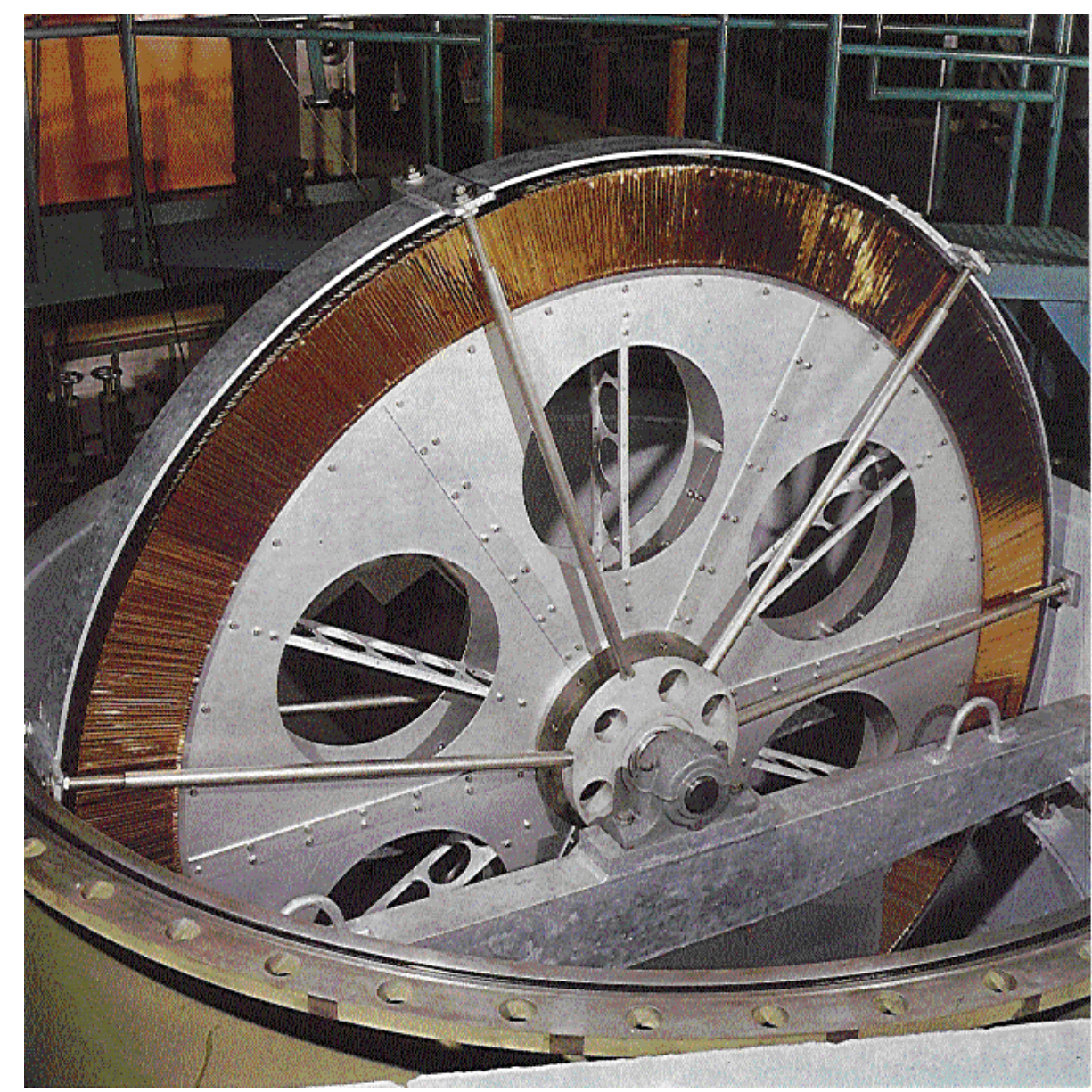

A. Steyerl et al., Phys. Lett. A 116 (1986) 347 


\section{Measuring the neutron EDM with bottled neutrons}

Procedure used in latest Sussex \RAL \ILL experiment:

(1) Fill bottle with spin-polarized ultra-cold neutrons (20s)

(2) Use rf field at Larmor frequency to rotate spins perpendicular to $E$ and $B(2 s)$

(3) Free precession in parallel \antiparallel E (10kV/cm) and B $(1 \mu \mathrm{T})$ for $130 \mathrm{~s}$

(4) Second pulse of $r f$ coherent with the first (2s)

(5) Open trap door and measure how many neutrons have spin-up and spin-down

(6) Reverse $E$ and do it again.... and again, and again, and again, and again, and again...

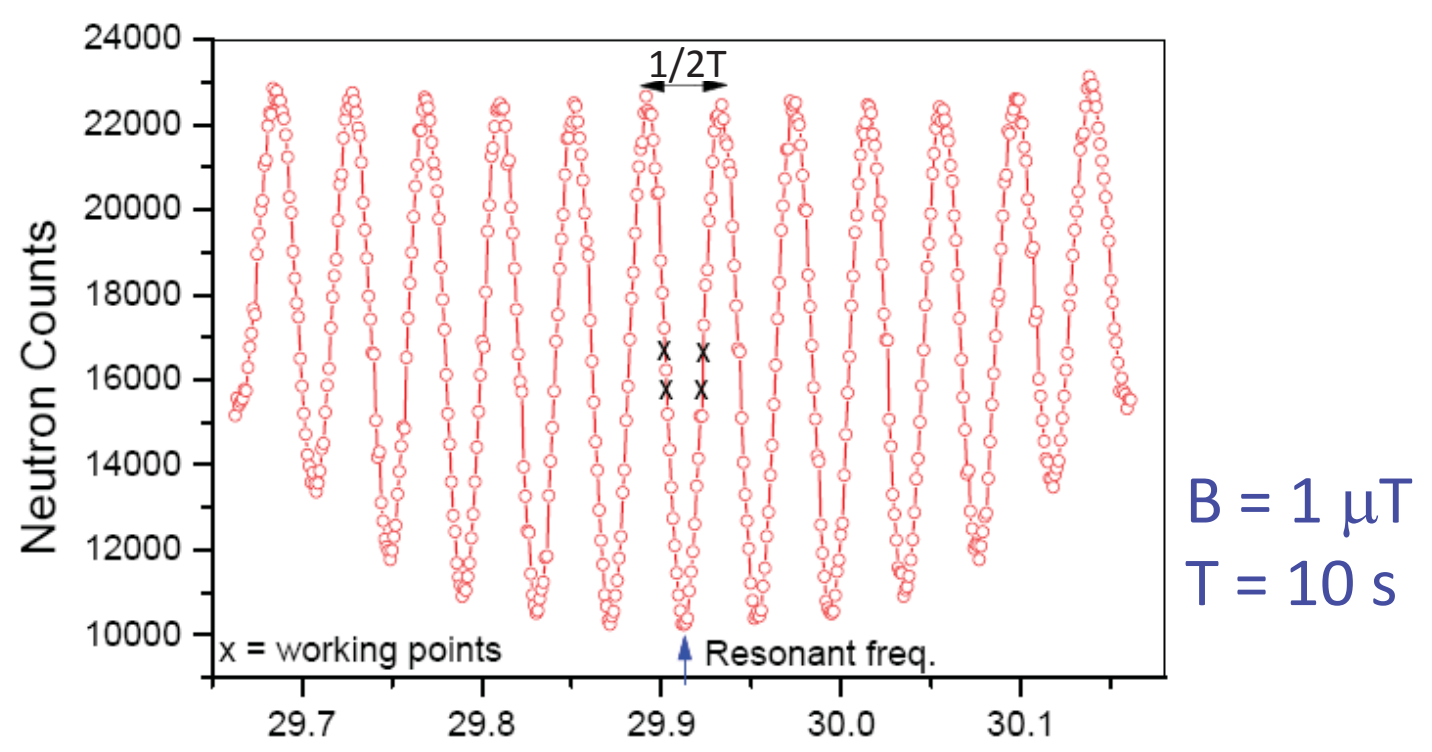

Applied Frequency $(\mathrm{Hz})$

Nucl. Instr. Meth. A 404, 381 (1998) 


\section{Measuring the neutron EDM with bottled neutrons}
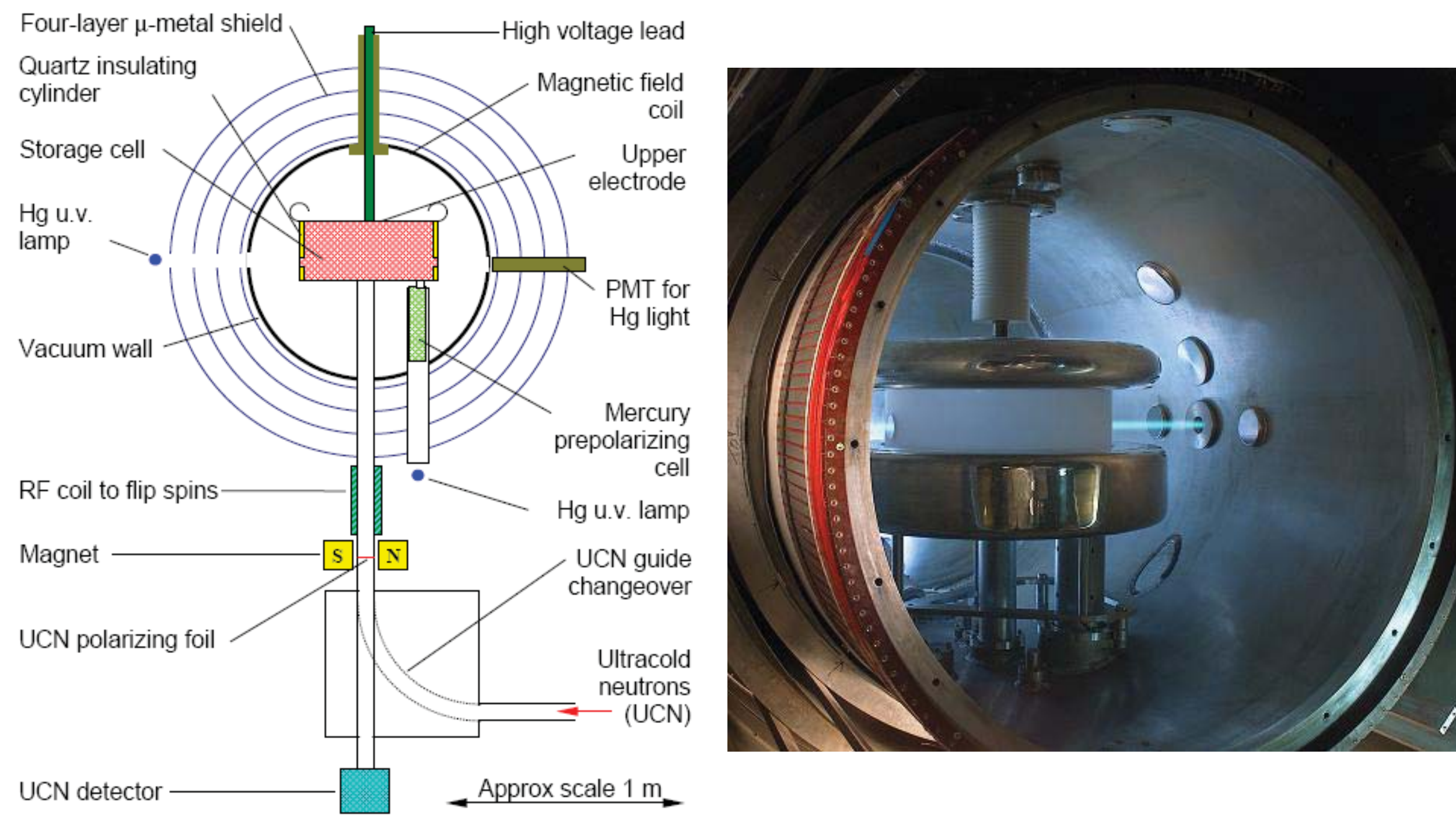

P. Harris, arXiv:0709.3100v3 


\section{Good magnetometry is essential}

Use spin precession of mercury atoms co-habiting in the bottle to determine the magnetic field and correct the neutron precession frequency

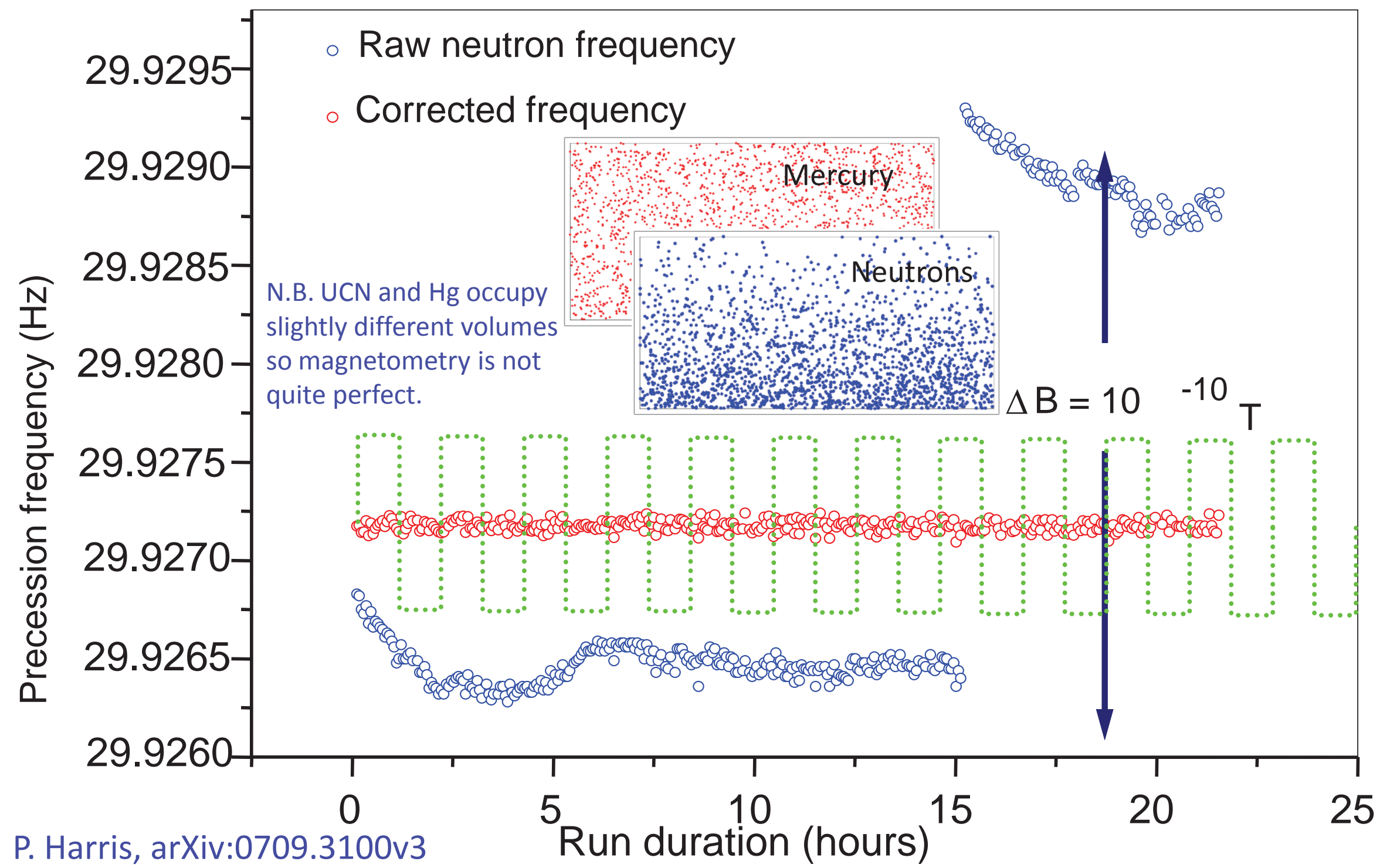




\section{Some systematic effects}

Magnetic fields correlated with E-reversal

Motional magnetic field, $\mathbf{v} \times \mathrm{E}$

Geometric phase 


\section{Measuring the electron EDM - an obvious problem!}
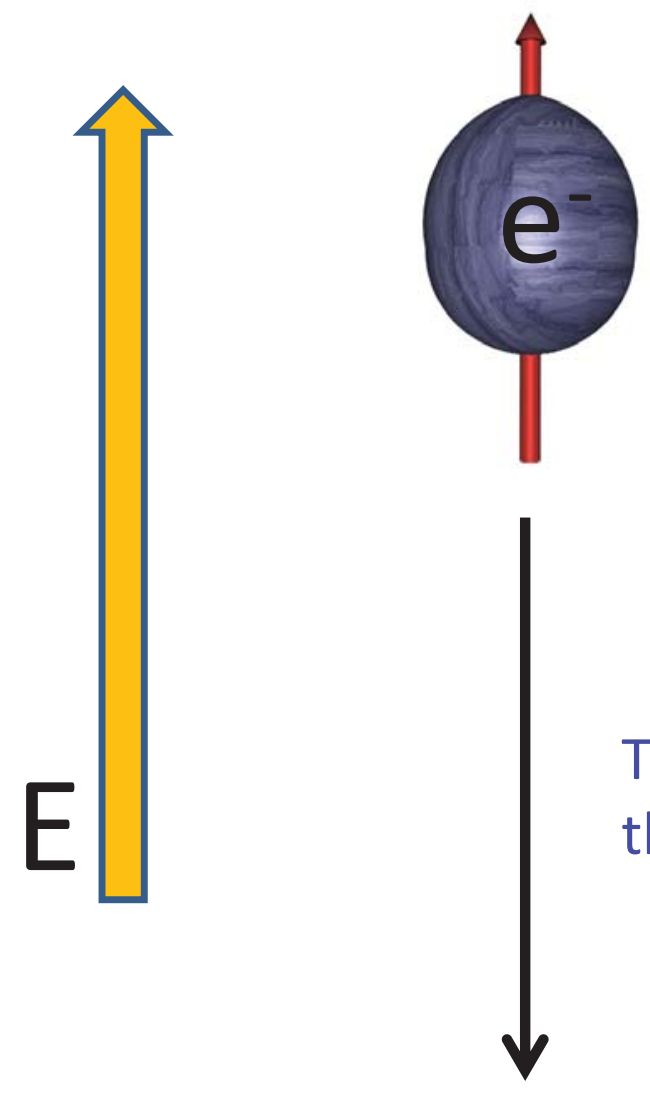

The electron accelerates away as soon as the electric field is applied

Solution: use a neutral system - an atom or a molecule 


\section{Schiff theorem}

When an electric field is applied to a system of non-relativistic point charges held together by electrostatic forces, the charges re-arrange so that the net electric field at every charge is zero
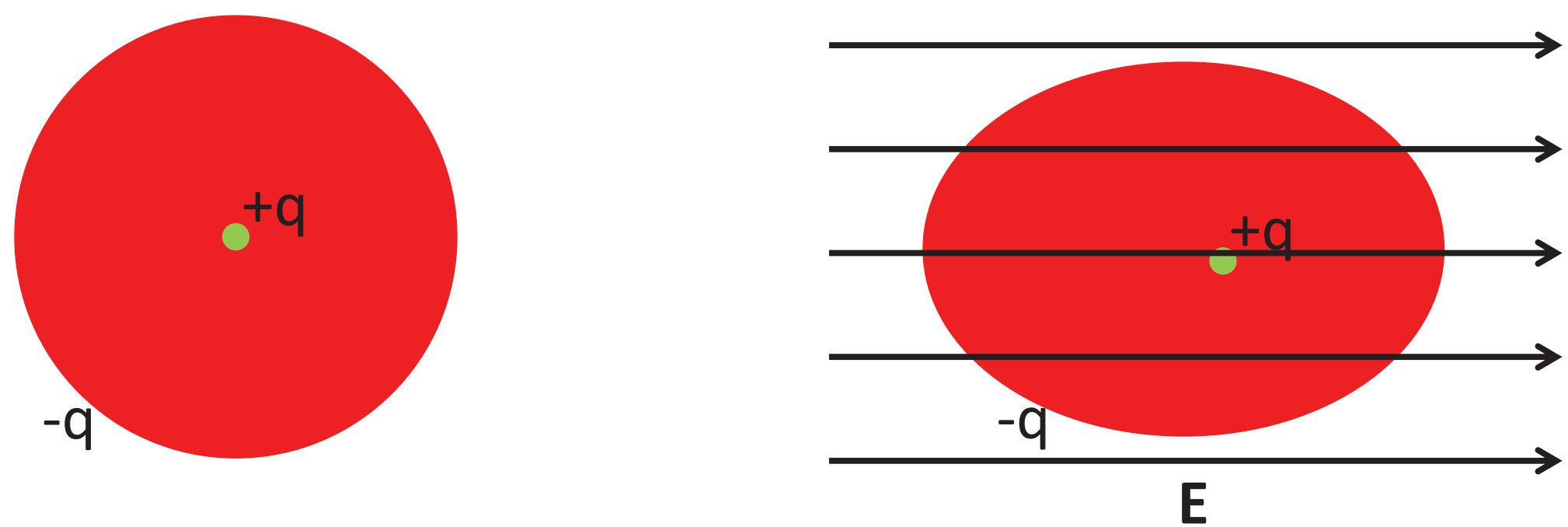

The EDM interaction energy will be zero even if the EDM of the constituents is non-zero!

Screening of the EDM 


\section{Evasion of the Schiff theorem}

The theorem applies to non-relativistic point particles held together only by electrostatic forces.

The electrons are relativistic, particularly for heavy atoms and molecules, so the screening is incomplete - in fact the electron EDM is not 'screened' but enhanced!

The nucleons are not point particles and are not held together only by electrostatic forces. So the screening is incomplete for the nucleons too. 


\section{Using atoms \& molecules to measure $d_{e}$}

For a free electron in an applied field $\underline{\mathbf{E}}$, expect an interaction energy $-\underline{\mathbf{d}}_{\mathrm{e}} \cdot \underline{\mathbf{E}}$

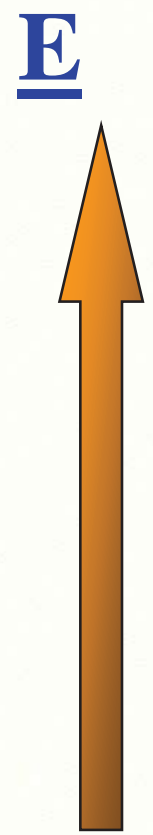

Electric

Field
Enhancement factor

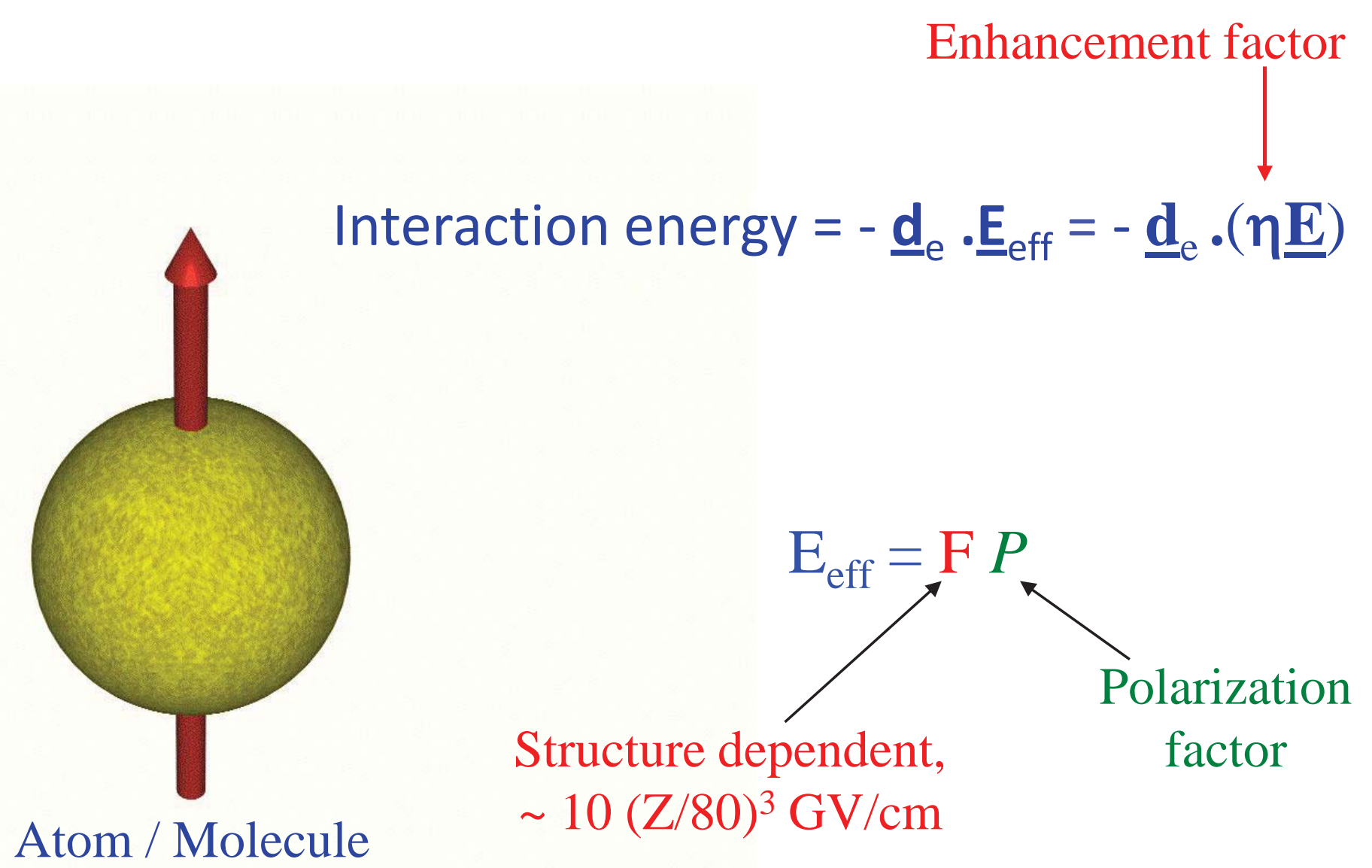

For more details, see E. A. Hinds, Physica Scripta T70, 34 (1997) 


\title{
Enhancement factors for some atoms
}

\author{
Enhancement factor, $\eta=d_{\text {atom }} / d_{\text {electron }}$
}

\begin{tabular}{|l|l|l|l|l|}
\hline Atom & $\mathrm{Z}$ & State & $\begin{array}{l}\text { Enhancement } \\
\text { factor }\end{array}$ & Experiments \\
\hline $\mathrm{Li}$ & 3 & $2^{2} \mathrm{~S}_{1 / 2}$ & 0.0043 & \\
\hline $\mathrm{Na}$ & 11 & $3^{2} \mathrm{~S}_{1 / 2}$ & 0.32 & \\
\hline $\mathrm{K}$ & 19 & $4{ }^{2} \mathrm{~S}_{1 / 2}$ & 2.4 & \\
\hline $\mathrm{Rb}$ & 37 & $5^{2} \mathrm{~S}_{1 / 2}$ & 25 & Early experiments. New experiments being developed \\
\hline $\mathrm{Cs}$ & 55 & $6^{2} \mathrm{~S}_{1 / 2}$ & 115 & with ultracold Cs (Texas \& Penn State) \\
\hline $\mathrm{Fr}$ & 87 & $7^{2} \mathrm{~S}_{1 / 2}$ & 1150 & $\begin{array}{l}\text { Radioactive. Experiment with ultracold }{ }^{210} \mathrm{Fr} \text { being } \\
\text { developed at RCNP, Osaka and TRIUMF, Vancouver }\end{array}$ \\
\hline $\mathrm{Tl}$ & 81 & $6^{2} \mathrm{P}_{1 / 2}$ & -585 & $\begin{array}{l}\text { Long-running experiment - world-leading results for } \\
\text { two decades. Now completed. }\end{array}$ \\
\hline
\end{tabular}




\section{The TI EDM experiment}

B.C. Regan, E.D. Commins, C.J. Schmidt and D. DeMille, PRL 88, 071805 (2002)

$\mathrm{Tl}$ - enhancement factor $\eta=-585$

polarise analyse

mun mon polarise

$4 \mathrm{Tl}$ atomic beams

\section{$1^{\text {st }}$ problem:}

motional interaction $\mu \cdot \mathbf{v} \times \mathbf{E} / \mathbf{c}^{2}$

\section{The solution:}

add 2 more $\mathrm{Tl}$ beams going down

$$
\mathrm{hf}_{ \pm}=\mu \mathrm{B} \quad \pm \mathbf{d} \eta \mathbf{E}
$$

\section{$2^{\text {nd }}$ problem:}

stray static magnetic fields

\section{The solution:}

Add $4 \mathrm{Na}$ beams for magnetometry 


\section{The TI EDM experiment}

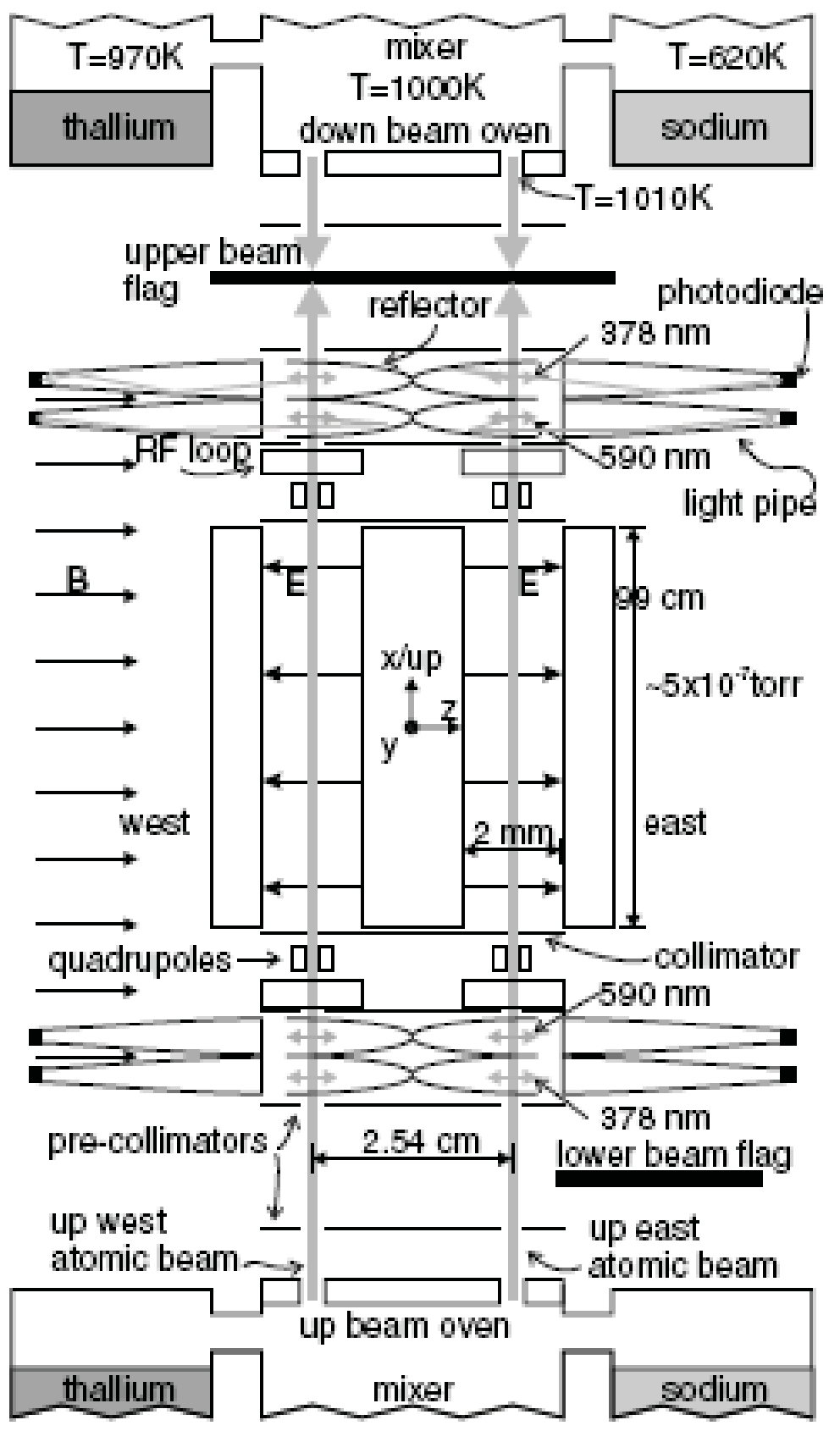

$$
\begin{gathered}
E=123 \mathrm{kV} / \mathrm{cm} \\
E_{\text {eff }}=72 \mathrm{MV} / \mathrm{cm} \\
T_{\text {coherence }}=2.3 \mathrm{~ms}
\end{gathered}
$$

Final result (2002) $\left|d_{e}\right|<1.6 \times 10^{-27}$ e.cm (90\% CL)

B.C. Regan, E.D. Commins, C.J. Schmidt \& D. DeMille, Phys. Rev. Lett. 88 , 071805(2002) 


\section{From atoms to molecules}
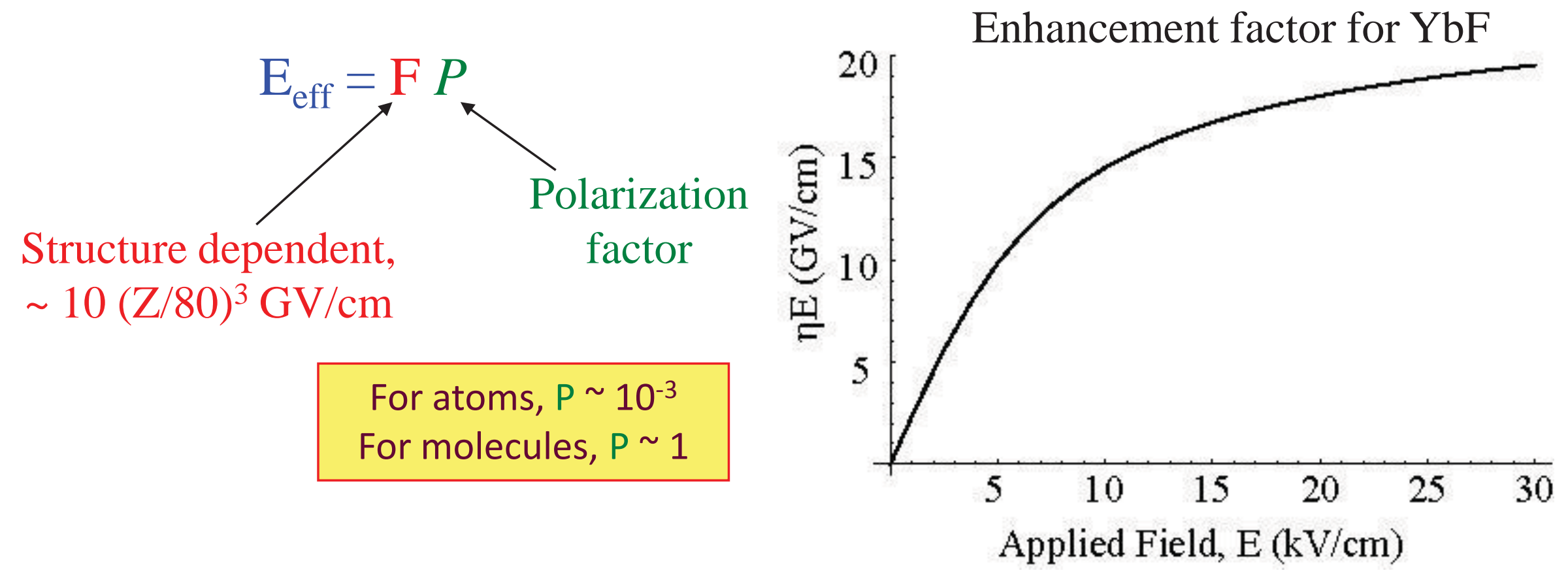

$>$ "Huge" edm interaction energy (10aeV, 2mHz, $\left.80 \mathrm{fm}^{-1}, 100 \mathrm{fK}\right)$

$>$ Less demanding magnetic field control $\left(\mathrm{d}_{\text {false }}=3 \times 10^{-27} \mathrm{e} . \mathrm{cm} / \mathrm{pT}\right)$

$>$ Insensitive to $\mathrm{B}$ perpendicular to $\mathrm{E}$ (suppressed by $10^{10}$ )

$>$ Thus, insensitive to motional-B $\left(\mathrm{B}_{\mathrm{mot}}=-\mathbf{v} \times \mathbf{E} / \mathrm{c}^{2}=10^{4} \mathrm{pT}\right)$ 


\section{With molecules, there's a price to pay}

- Molecules are far more difficult to produce and detect than atoms

- They have a much more complicated structure \spectrum

- Often not much is known - so you have to do a lot of preliminary spectroscopy

Sauer, Wang, and Hinds: Spectroscopy of ${ }^{174} \mathrm{YbF}$

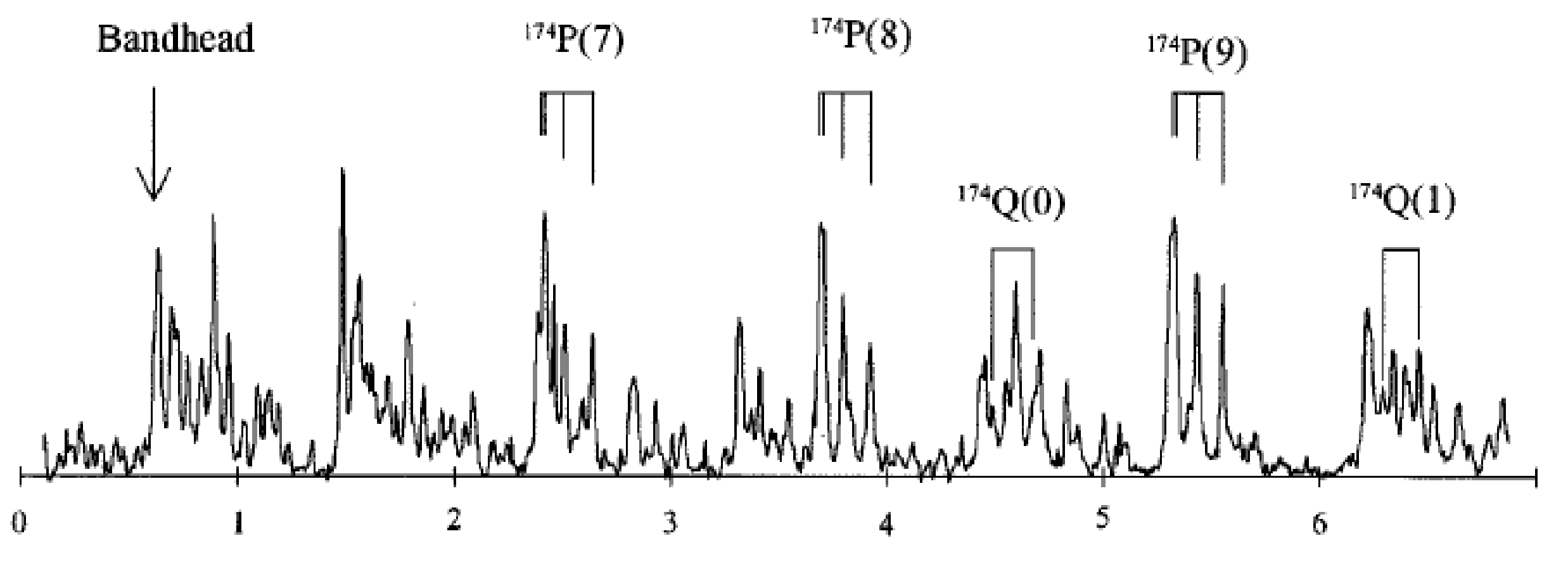

Relative laser frequency $(\mathrm{GHz})$

J. Chem. Phys 105, 7412 (1996) 


\section{Relevant energy levels in the YbF molecule}

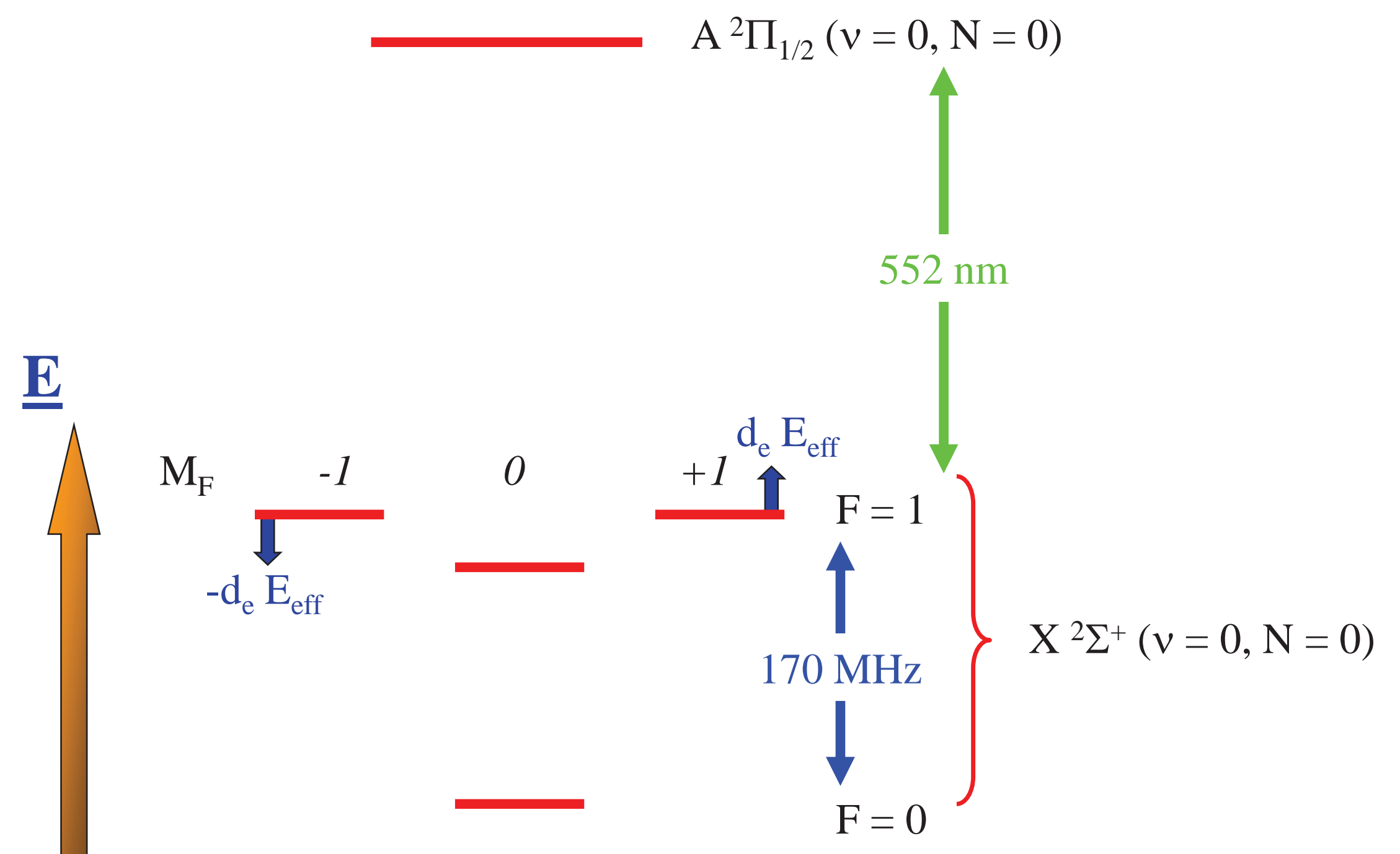

Electric Measure the splitting $2 \mathrm{~d}_{\mathrm{e}} \mathrm{E}_{\text {eff }}$ between the $\mathrm{M}_{\mathrm{F}}=+1$ and $\mathrm{M}_{\mathrm{F}}=-1$ levels Field 


\section{The YbF EDM experiment - measurement scheme}

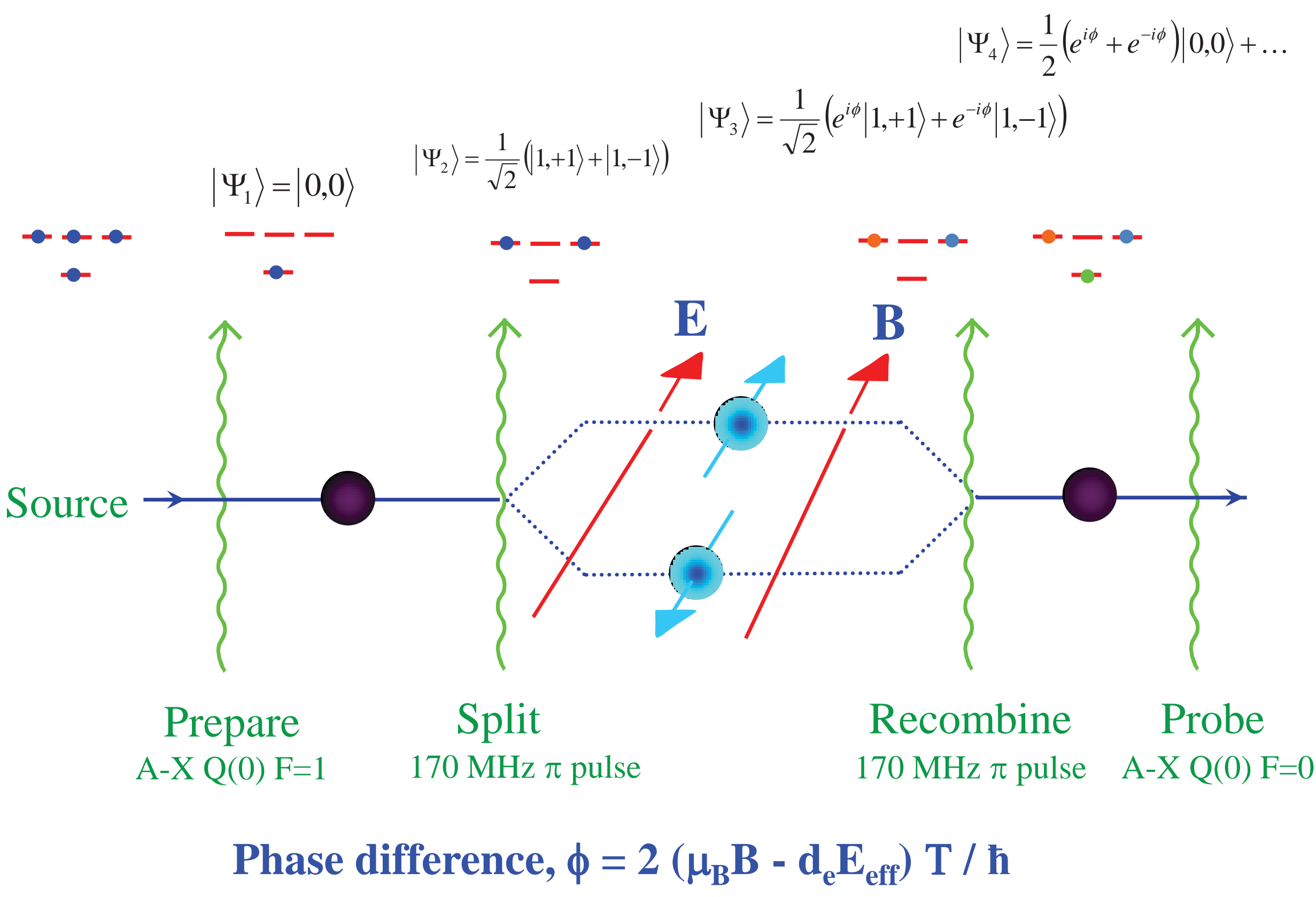




\section{The YbF EDM experiment - schematic}

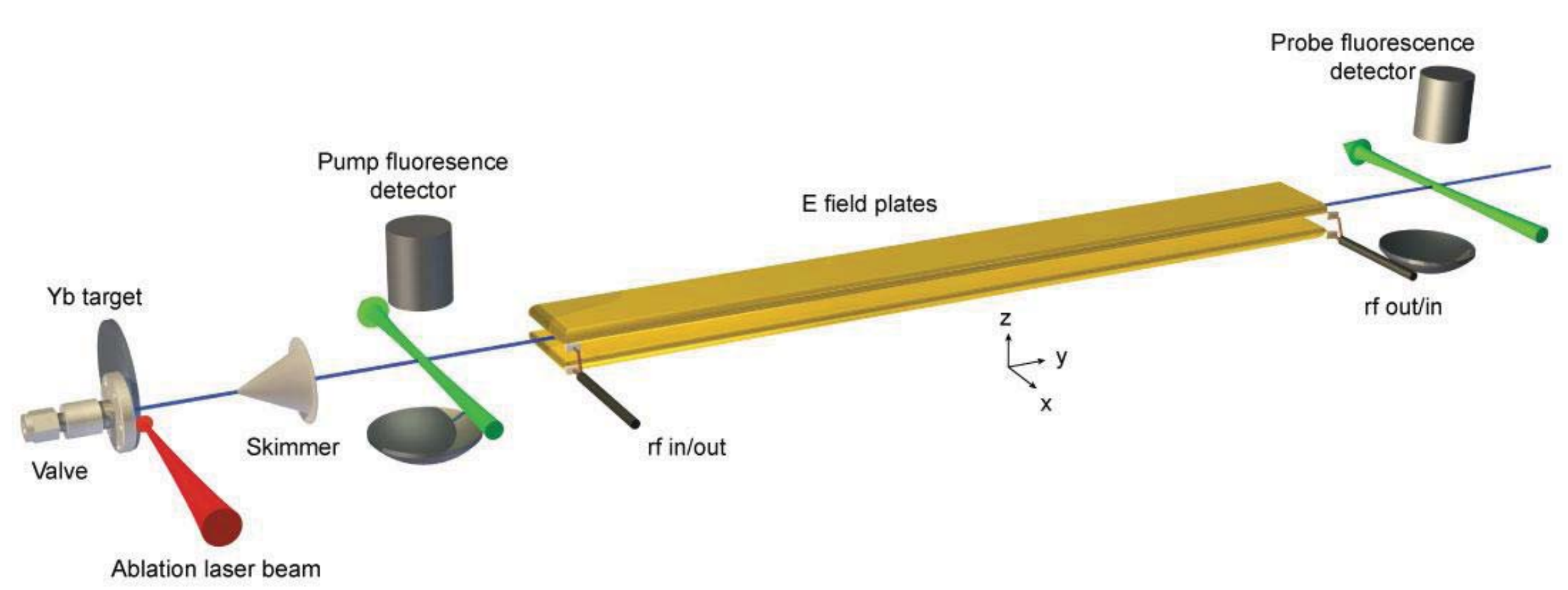

J. J. Hudson, D. M. Kara, I. J. Smallman, B. E. Sauer, M. R. Tarbutt \& E. A. Hinds, Nature 473, 493 (2011) 


\section{Measurement scheme continued}

$$
\left|\Psi_{4}\right\rangle=\frac{1}{2}\left(e^{i \phi}+e^{-i \phi}\right)|0,0\rangle+\ldots
$$

The signal is proportional to $\left|\left\langle 0,0 \mid \Psi_{4}\right\rangle\right|^{2}$

$$
\text { Signal } \alpha \operatorname{Cos}^{2}[\phi / 2]=\operatorname{Cos}^{2}\left[\frac{\mu_{\mathrm{B}} \mathrm{B}-\eta \mathrm{d}_{\mathrm{e}} \mathrm{E}}{\hbar} \mathrm{T}\right]
$$

Measured $\mathrm{F}=0$ population versus applied magnetic field

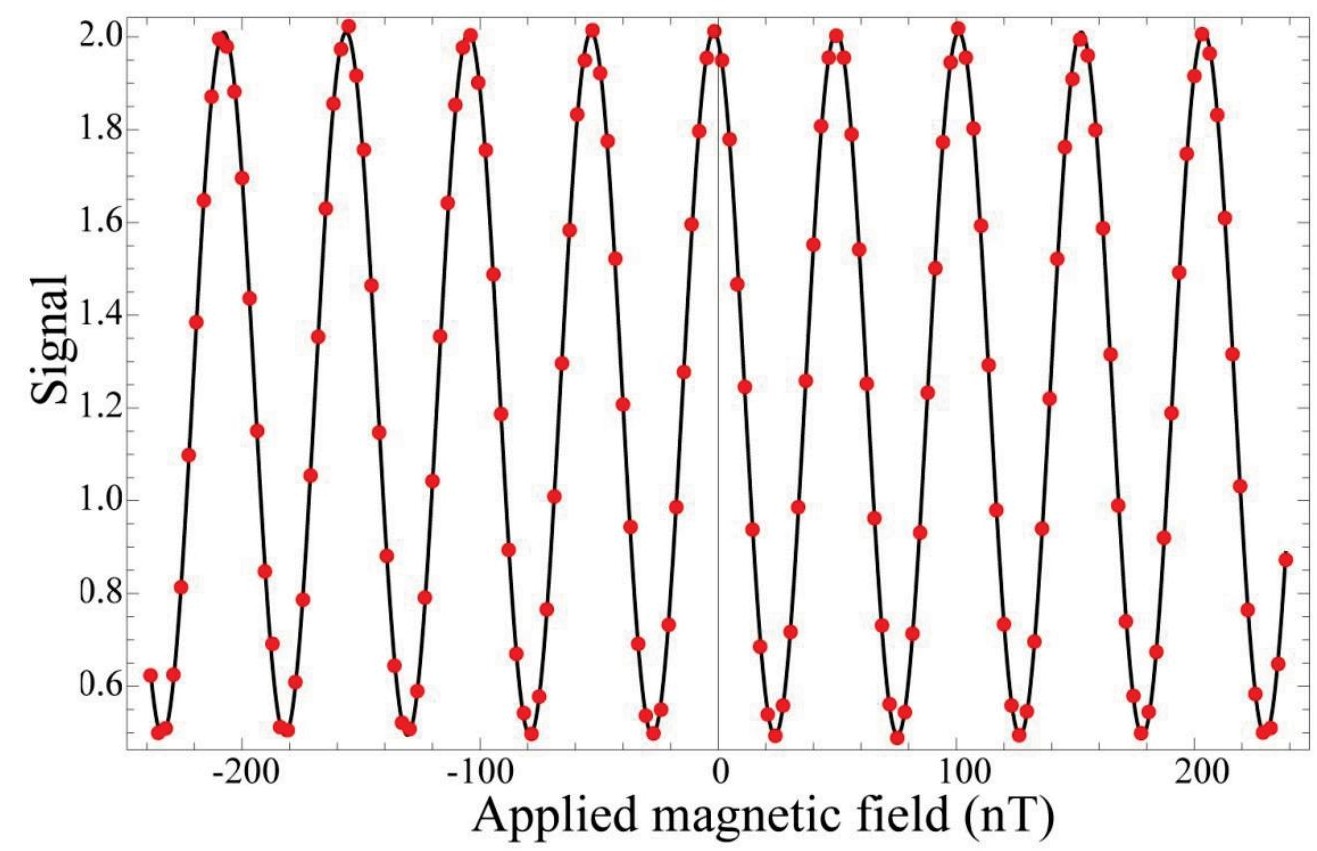




\section{Measure the change in signal when $\mathrm{E}$ is reversed}

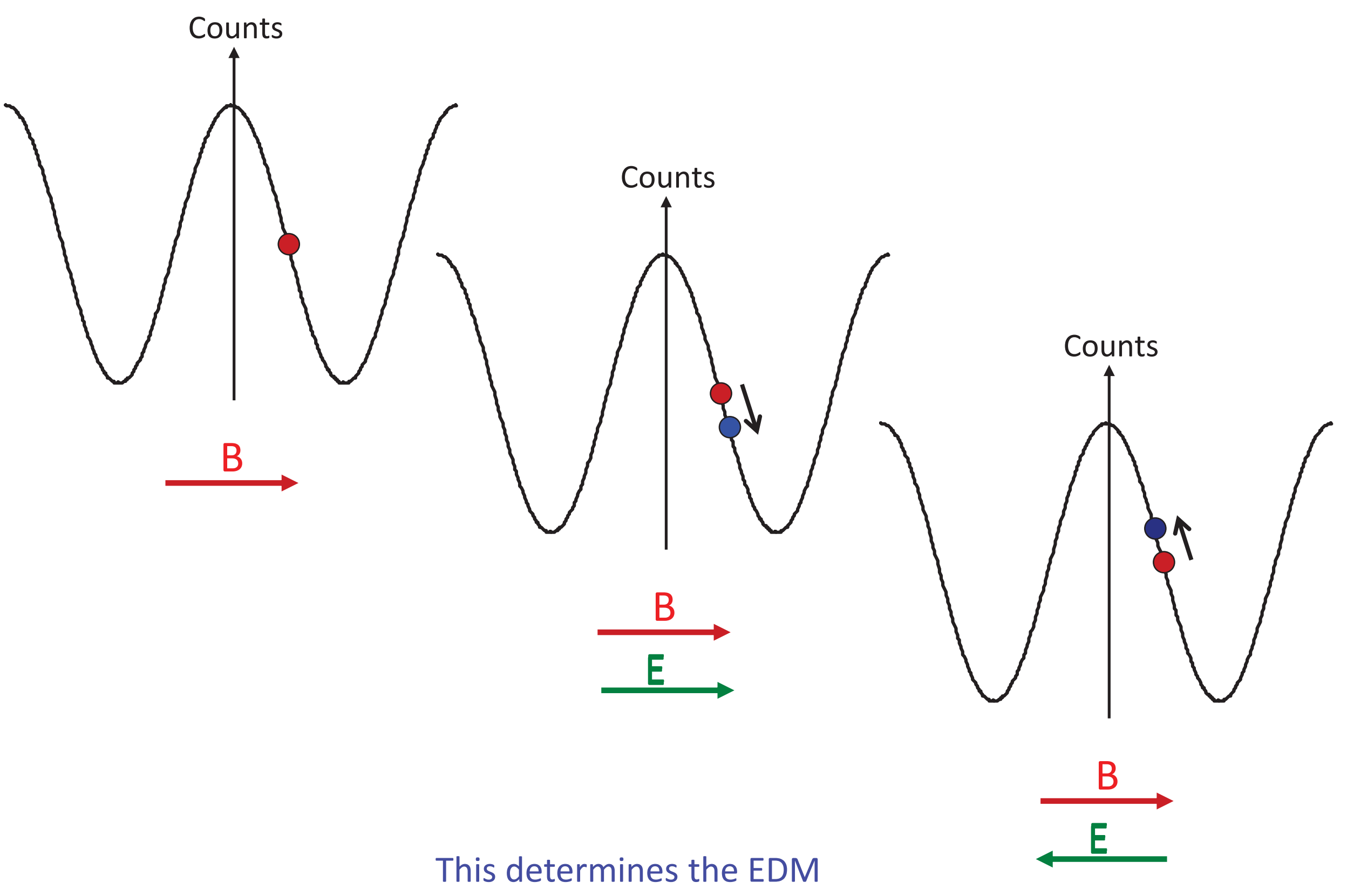




\section{Modulate everything}

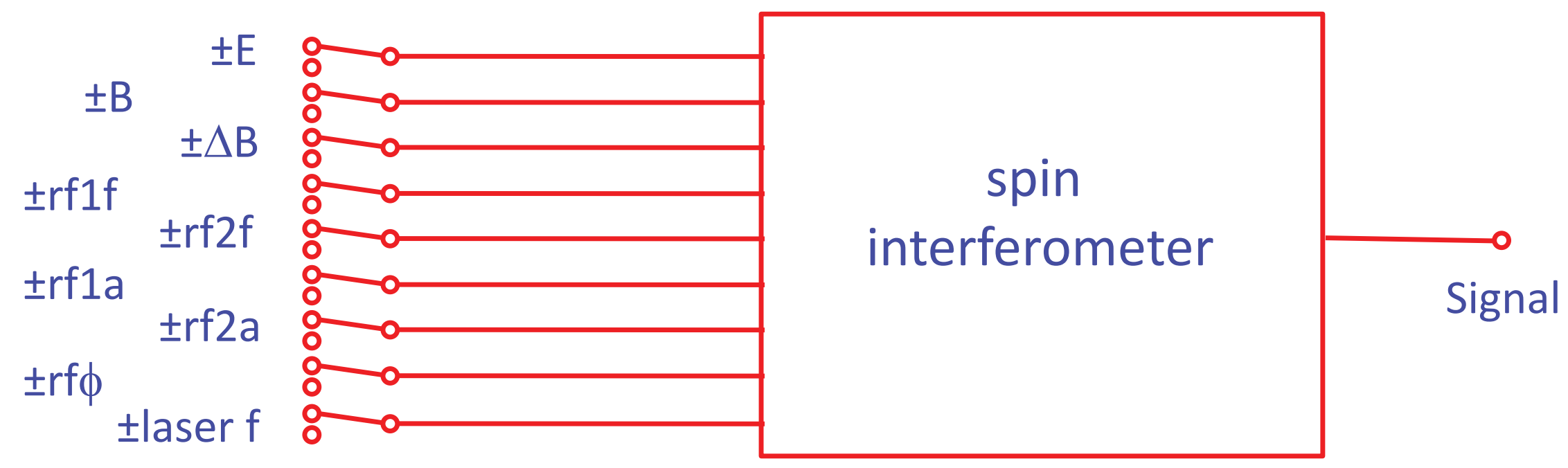

9 switches:

512 possible correlations

$>$ The EDM is the signal correlated with the sign of E.B (N.B. Blind in the analysis)

$>$ Study all the other 511 correlations in detail 


\section{Result (2011)}

$>6194$ measurements of the EDM, each derived from 4096 beam pulses

$>$ Each measurement takes 6 minutes

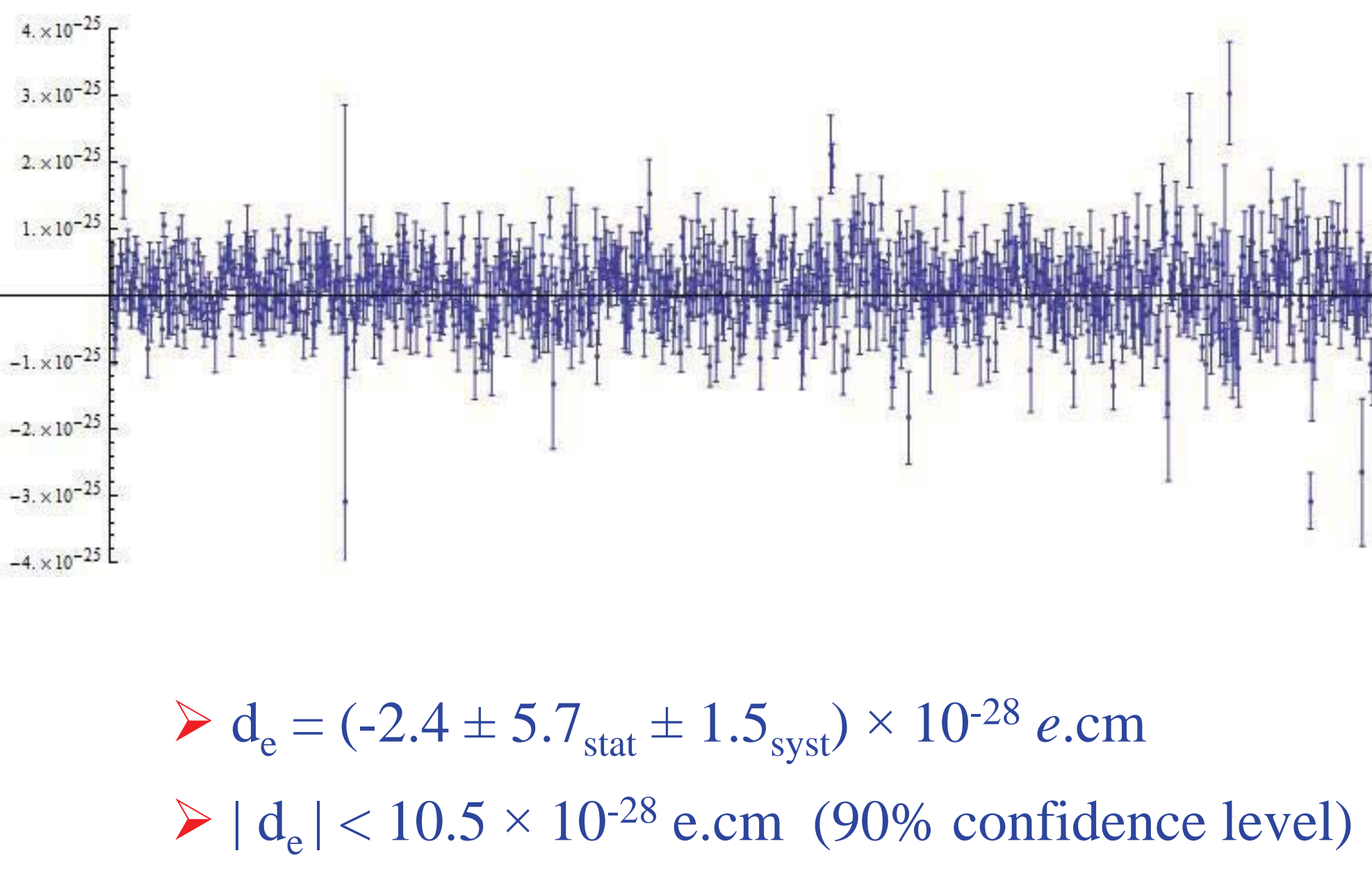

J. J. Hudson, D. M. Kara, I. J. Smallman, B. E. Sauer, M. R. Tarbutt \& E. A. Hinds, Nature 473, 493 (2011) 


\section{Controlling systematics...}

$>$ Use the molecules to make maps of the electric, magnetic and rf fields

Electric field variation along plates
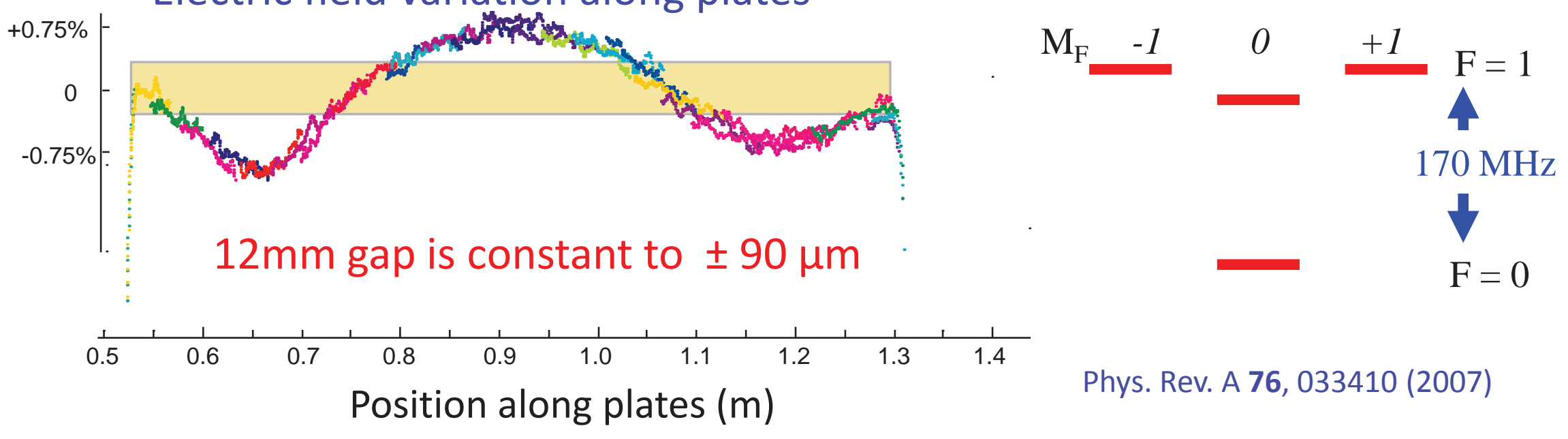

$>$ Use the molecules to determine how well the electric field reverses

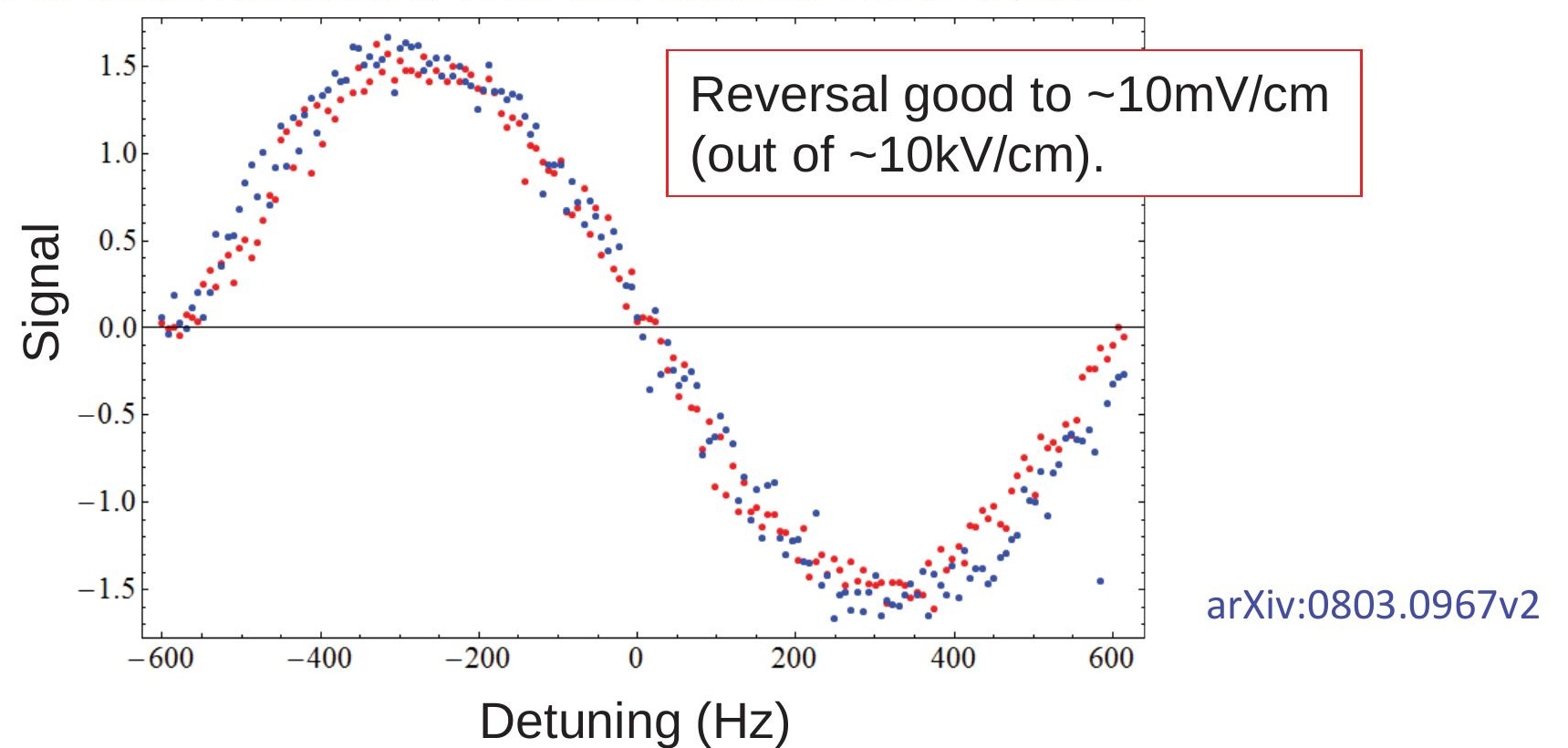




\section{Controlling systematics...}

$>$ Look for suspicious channels - ones that should be zero but are not

> Take data with imperfections deliberately emphasized (e.g. E asymmetry)

$>$ Simultaneous data from pump PMT, 4 separate magnetometers, 2 leakage current monitors, a battery and a short-circuit.

$>$ Divide molecular pulses into early-half and late-half. Check for consistency.

$>$ Manual reversals of E-direction, B-direction and rf propagation direction

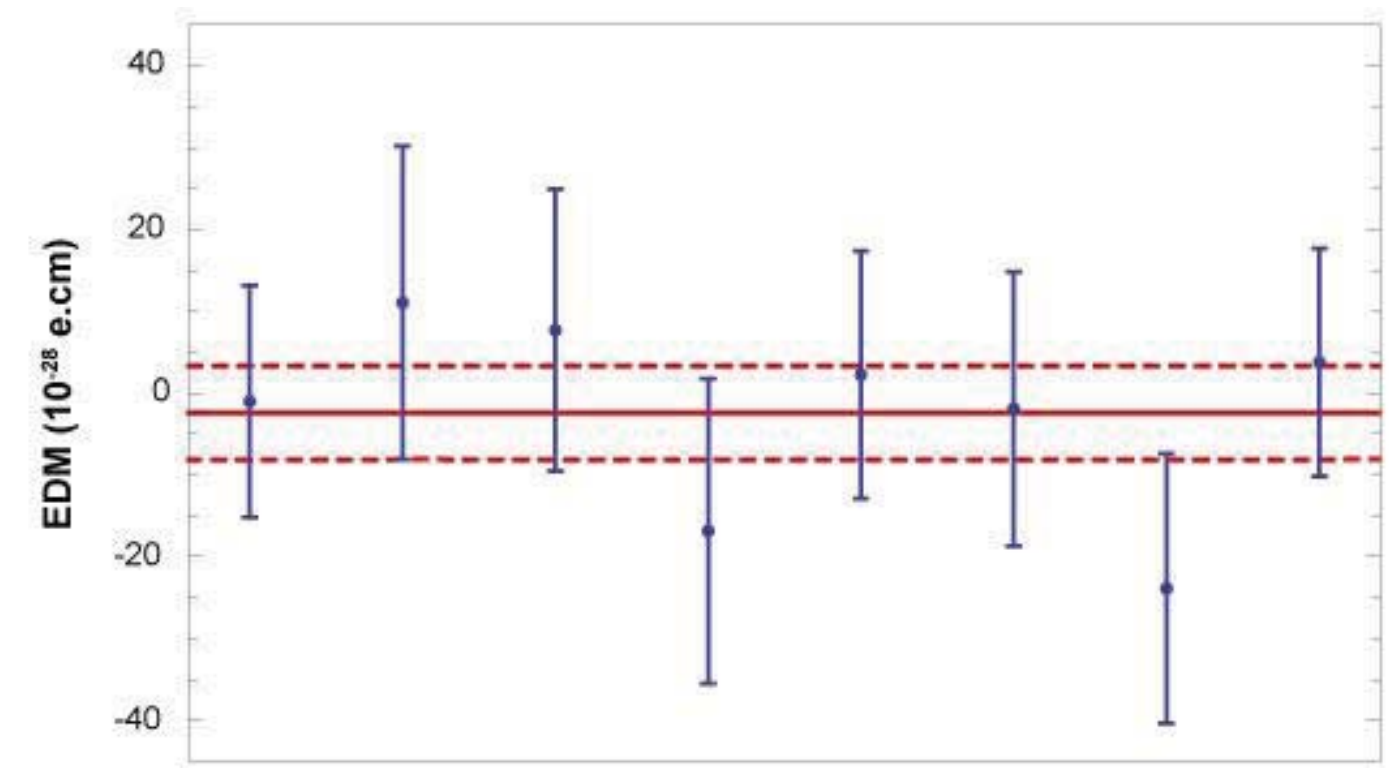




\section{Correcting a systematic error}
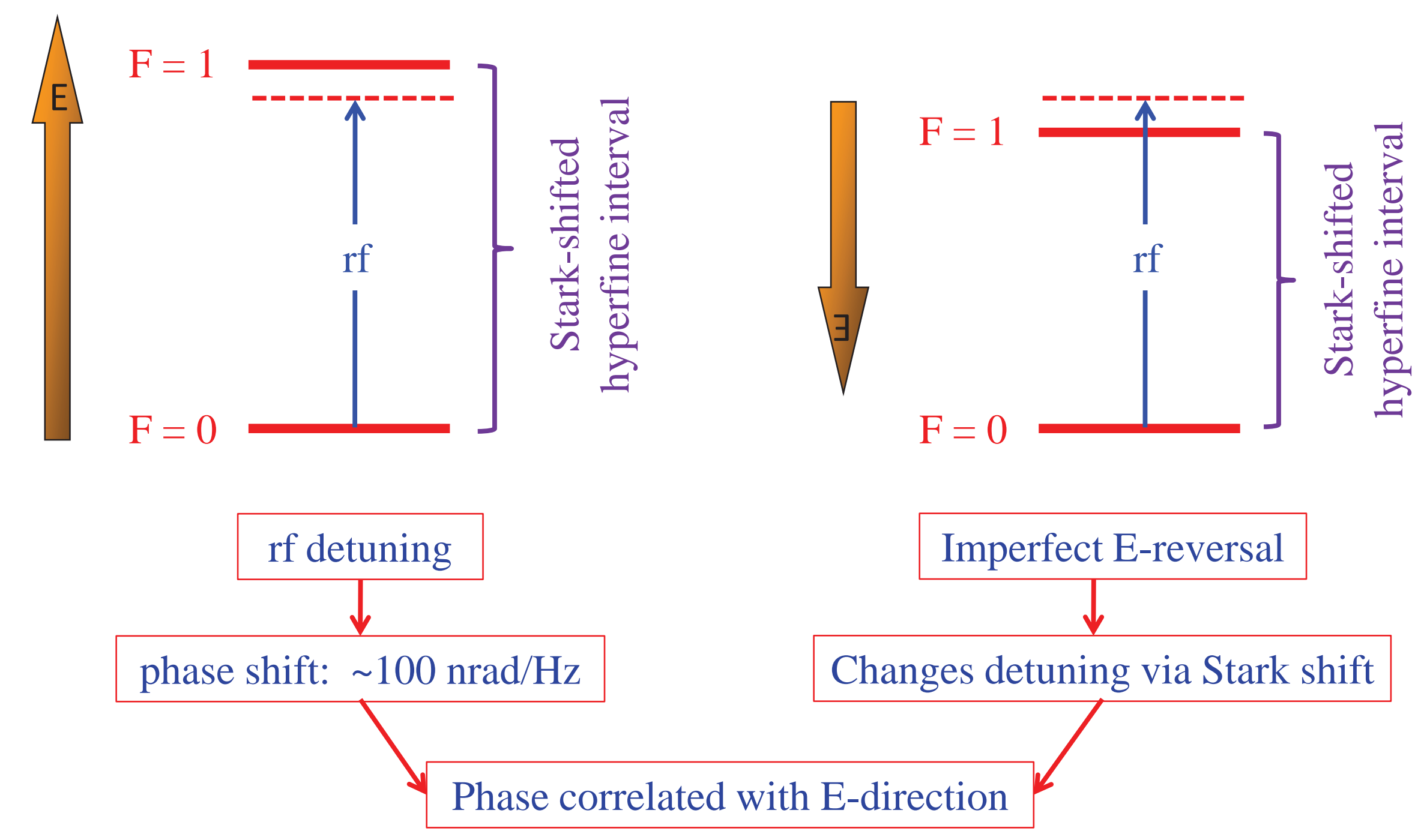

Correction to EDM: $(5.5 \pm 1.5) \times 10^{-28}$ e.cm 


\section{Systematic uncertainties}

\begin{tabular}{|c|c|}
\hline Effect & $\begin{array}{c}\text { Systematic uncertainty } \\
\left(10^{-28} \text { e.cm }\right)\end{array}$ \\
\hline Electric field asymmetry & 1.1 \\
\hline Electric potential asymmetry & 0.1 \\
\hline Residual RF1 correlation & 1.0 \\
\hline Geometric phase & 0.03 \\
\hline Leakage currents & 0.2 \\
\hline Shield magnetization & 0.25 \\
\hline Motional magnetic field & 0.0005 \\
\hline
\end{tabular}




\section{Some new methods for future experiments}

Cryogenic sources of molecules

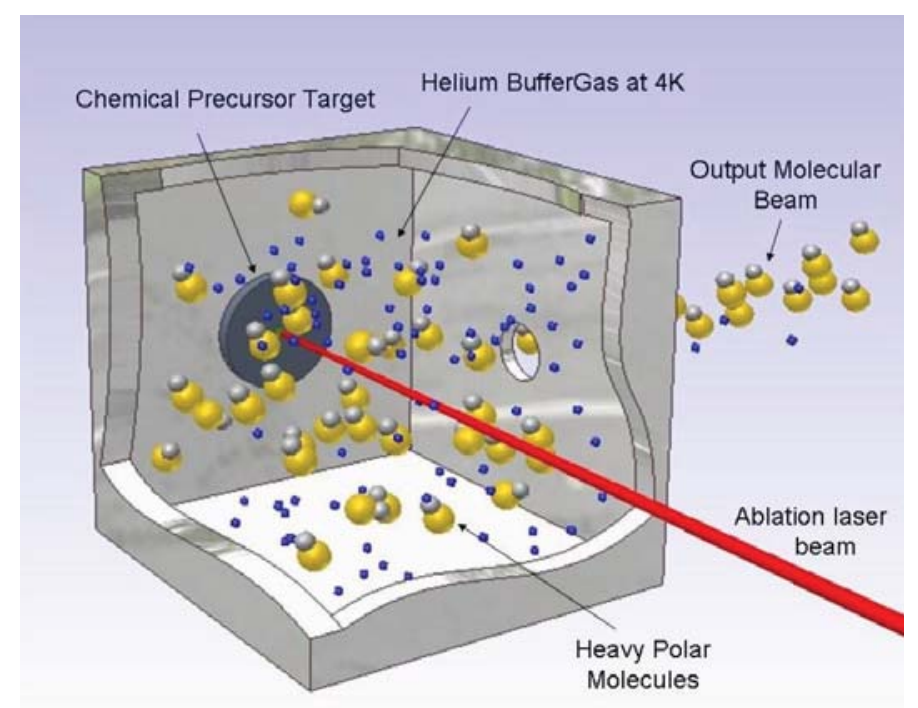

e.g. H.-I. Lu, J. Rasmussen, M. J. Wright, D. Patterson and J. M. Doyle, Phys. Chem. Chem. Phys. 13, 18986 (2011)
Laser cooling of molecules

See E. S. Shuman, J. F. Barry and D. DeMille, Nature 467, 820 (2010)

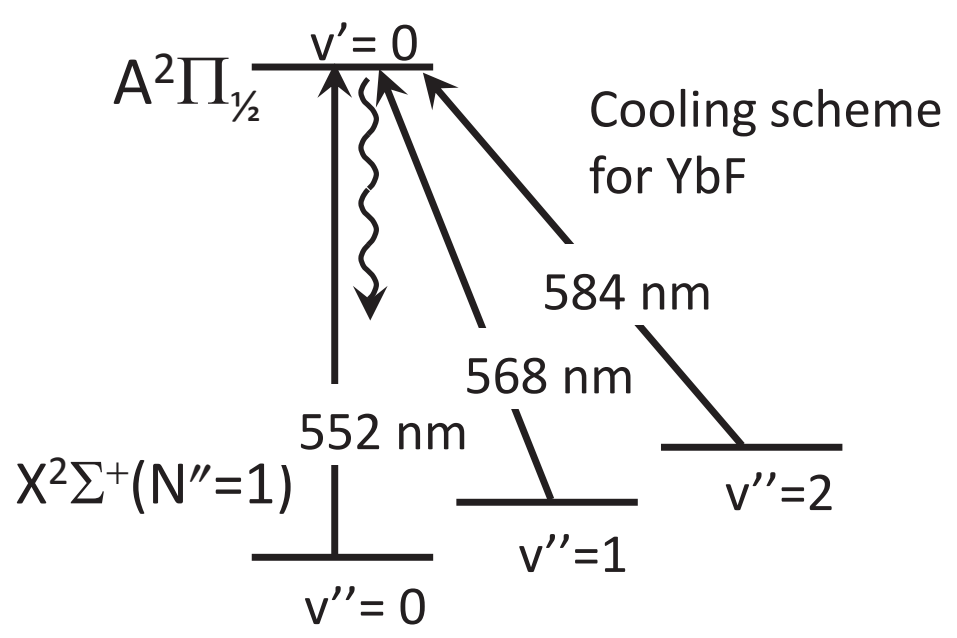

Laser-cooled molecular fountain:

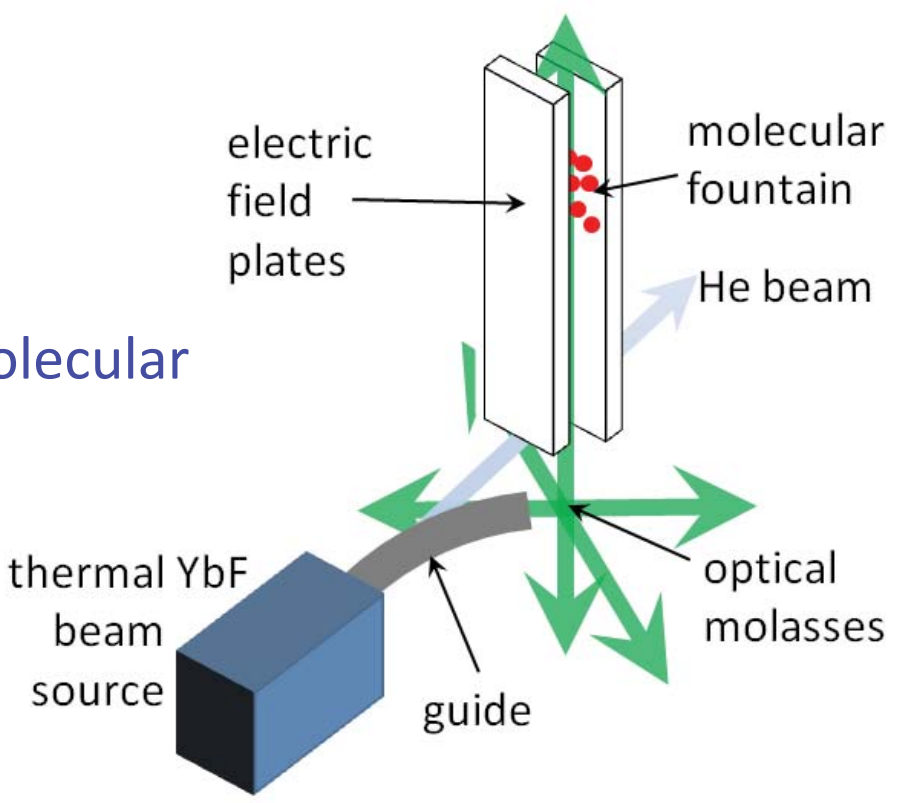




\section{Some ongoing and future electron EDM experiments}

\begin{tabular}{|c|c|c|c|}
\hline System & References & $\begin{array}{l}E_{\text {eff }} \\
(\mathrm{GV} / \mathrm{cm})\end{array}$ & Features \\
\hline YbF & Nature 473, 493 (2011). & 26 & $\begin{array}{l}\text { Current best limit. New measurements and upgrades in } \\
\text { progress. }\end{array}$ \\
\hline $\mathrm{PbO}$ & Phys. Rev. Lett. 92133007 (2004). & 26 & $\begin{array}{l}\text { Cell expt. Fully polarized at low field. Internal co- } \\
\text { magnetometer. Metastable state ( } 82 \mu \mathrm{s}) \text {. }\end{array}$ \\
\hline ThO & J. Phys. B 43074007 (2010). & 104 & $\begin{array}{l}\text { Cryogenic beam expt. Fully polarized at low field. Internal } \\
\text { co-magnetometer. Small g-factor. Metastable state ( } 2 \mathrm{~ms}) \text {. }\end{array}$ \\
\hline WC & J. Mod. Optics 56, 2005 (2009). & 54 & $\begin{array}{l}\text { Ground-state. Fully polarized at low field. Internal co- } \\
\text { magnetometer. Small g-factor. }\end{array}$ \\
\hline $\mathrm{PbF}$ & Phys. Rev. A 73, 034102 (2006). & -29 & Zero g-factor. \\
\hline Cs & $\begin{array}{l}\text { Phys. Rev. A 63, } 033401 \text { (2001); Bull. } \\
\text { Am. Phys. Soc. APR03, J1.008 (2003). }\end{array}$ & 0.01 & $\begin{array}{l}\text { New experiments with optically trapped ultracold Cs } \\
\text { (Penn State and U. Texas) }\end{array}$ \\
\hline $\mathrm{Fr}$ & $\begin{array}{l}\text { http://www.rcnp.osaka- } \\
\text { u.ac.jp/ sakemi/EDM/edmRiken.pdf; } \\
\underline{\text { http://eedm.info/index.html. }}\end{array}$ & 0.1 & $\begin{array}{l}\text { Radioactive. Experiment with ultracold }{ }^{210} \mathrm{Fr} \text { being } \\
\text { developed (RCNP, Osaka and TRIUMF, Vancouver) }\end{array}$ \\
\hline $\mathrm{HfF}^{+}$ & J. Mol. Spectrosc. 270,1 (2011). & 18 & $\begin{array}{l}\text { lon trap experiment with rotating electric and magnetic } \\
\text { fields. }\end{array}$ \\
\hline Solids & $\begin{array}{l}\text { Phys. Rev. A 66, } 022109 \text { (2002); Phys. } \\
\text { Rev. Lett. 95, } 253004 \text { (2005); } \\
\text { Mod. Phys. Lett. A 19, } 1235 \text { (2004) }\end{array}$ & 0.00003 & Lots of electrons. Difficult to control systematic effects. \\
\hline
\end{tabular}




\section{Mercury EDM experiment}

\section{${ }^{199} \mathrm{Hg}$ EDM Experimental Setup}
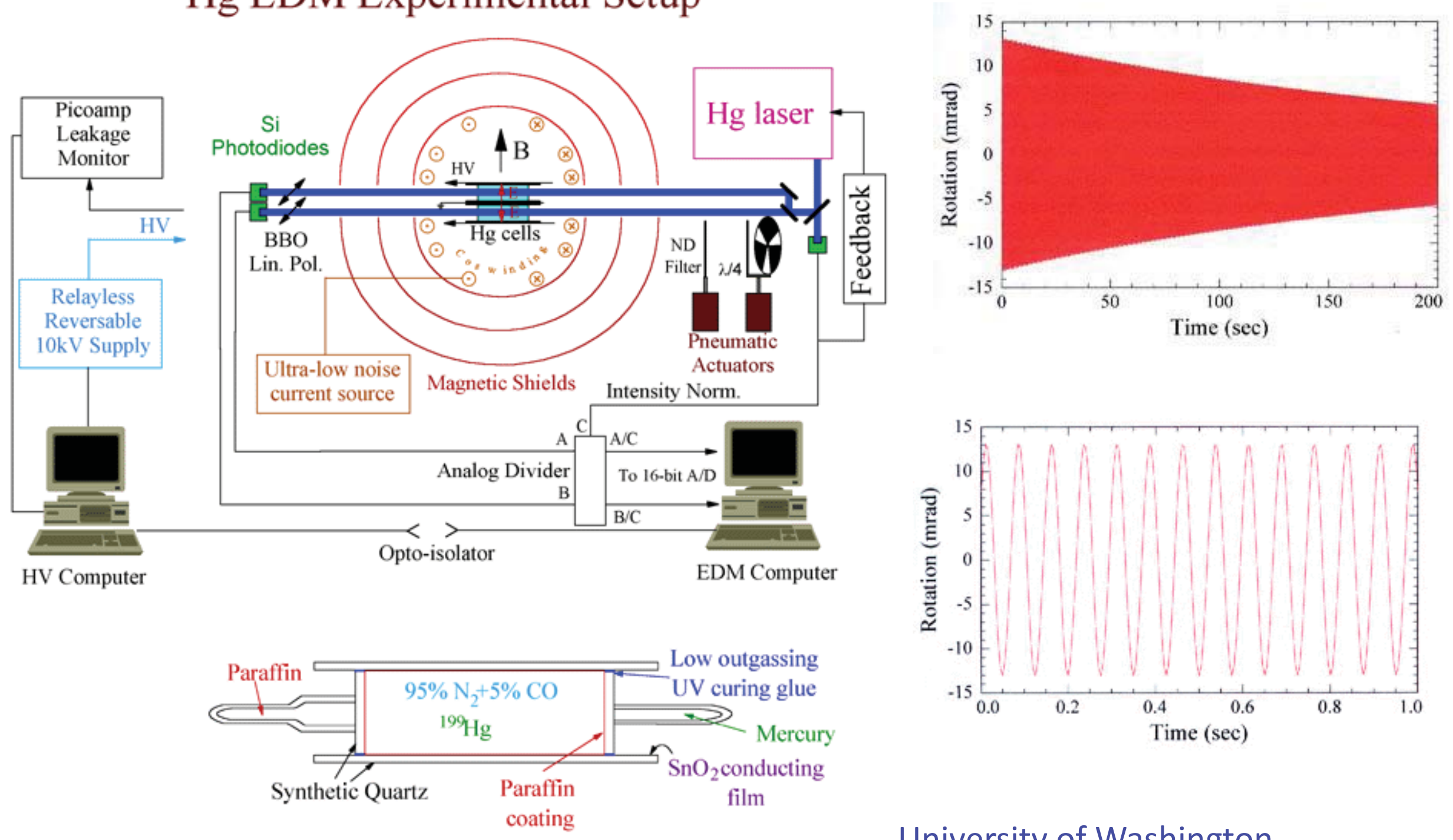

University of Washington

Phys. Rev. Lett. 102, 101601 (2009) 


\section{Mercury EDM experiment}

Result: $\quad\left|d\left({ }^{199} \mathrm{Hg}\right)\right|<3.1 \times 10^{-29}$ e.cm $(95 \% \mathrm{CL})$

Phys. Rev. Lett. 102, 101601 (2009)

The most precise measurement of the EDM of anything

\begin{tabular}{|c|c|c|c|c|}
\hline Parameter & ${ }^{199} \mathrm{Hg}$ bound & $\mathrm{Hg}$ theory & Best alternate limit & \\
\hline $\begin{array}{l}\tilde{d}_{q}(\mathrm{~cm})^{\mathrm{a}} \\
d_{n}(e \mathrm{~cm})\end{array}$ & $\begin{array}{r}6 \times 10^{-27} \\
7.9 \times 10^{-25}\end{array}$ & $\begin{array}{l}{[15]} \\
{[16]}\end{array}$ & $\begin{aligned} \mathrm{n} & : 3 \times 10^{-26}[3] \\
\mathrm{T} 1 \mathrm{~F}: & 6 \times 10^{-23}[17]\end{aligned}$ & $|d(p)|<7.9 \times 10^{-25}$ e.cm \\
\hline$C_{S}$ & $5.2 \times 10^{-8}$ & [18] & Tl: $2.4 \times 10^{-7}[19]$ & \\
\hline$C_{P}$ & $5.1 \times 10^{-7}$ & [18] & TIF: $3 \times 10^{-4}[1]$ & $|d(n)|<5.8 \times 10^{-26}$ e.cm \\
\hline$C_{T}$ & $1.5 \times 10^{-9}$ & [18] & $\mathrm{TlF}: 4.5 \times 10^{-7}[1]$ & \\
\hline $\bar{\theta}_{\mathrm{OCD}}$ & $3 \times 10^{-10}$ & [20] & $\mathrm{n}: 1 \times 10^{-10}[3]$ & \\
\hline$d_{n}(e \mathrm{~cm})$ & $5.8 \times 10^{-26}$ & [16] & $\mathrm{n}: 2.9 \times 10^{-26}$ & \\
\hline$d_{e}(e \mathrm{~cm})$ & $3 \times 10^{-27}$ & {$[21,22]$} & Tl: $1.6 \times 10^{-27}[18]$ & \\
\hline
\end{tabular}




\section{Consequences of electron EDM measurements}

Electron EDM from a one-loop diagram in supersymmetry is

$d_{e} \sim\left(\frac{\alpha}{\pi}\right) \frac{m_{e}}{\Lambda^{2}} \sin \phi_{\mathrm{CP}}$
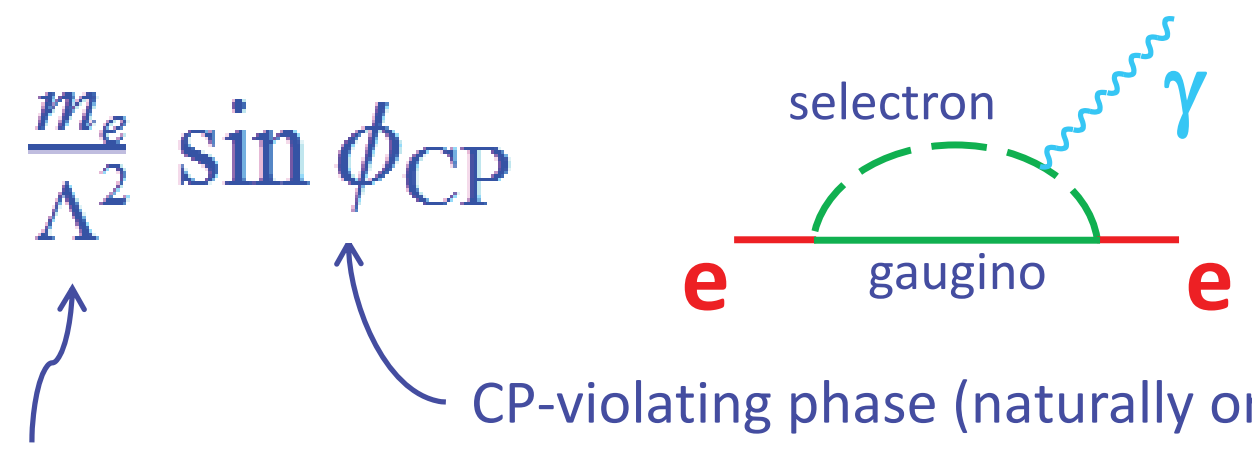

Scale of SUSY breaking 200GeV

$$
d_{e} \sim 5 \times 10^{-25} \text { e.cm naturally }
$$

"Natural" SUSY EDM is 500 times too big

$$
\Lambda>4 \mathrm{TeV} \text { ?? }
$$

$$
\phi_{\mathrm{CP}}<10^{-3} \text { ?? }
$$




\section{Consequences of electron EDM measurements}

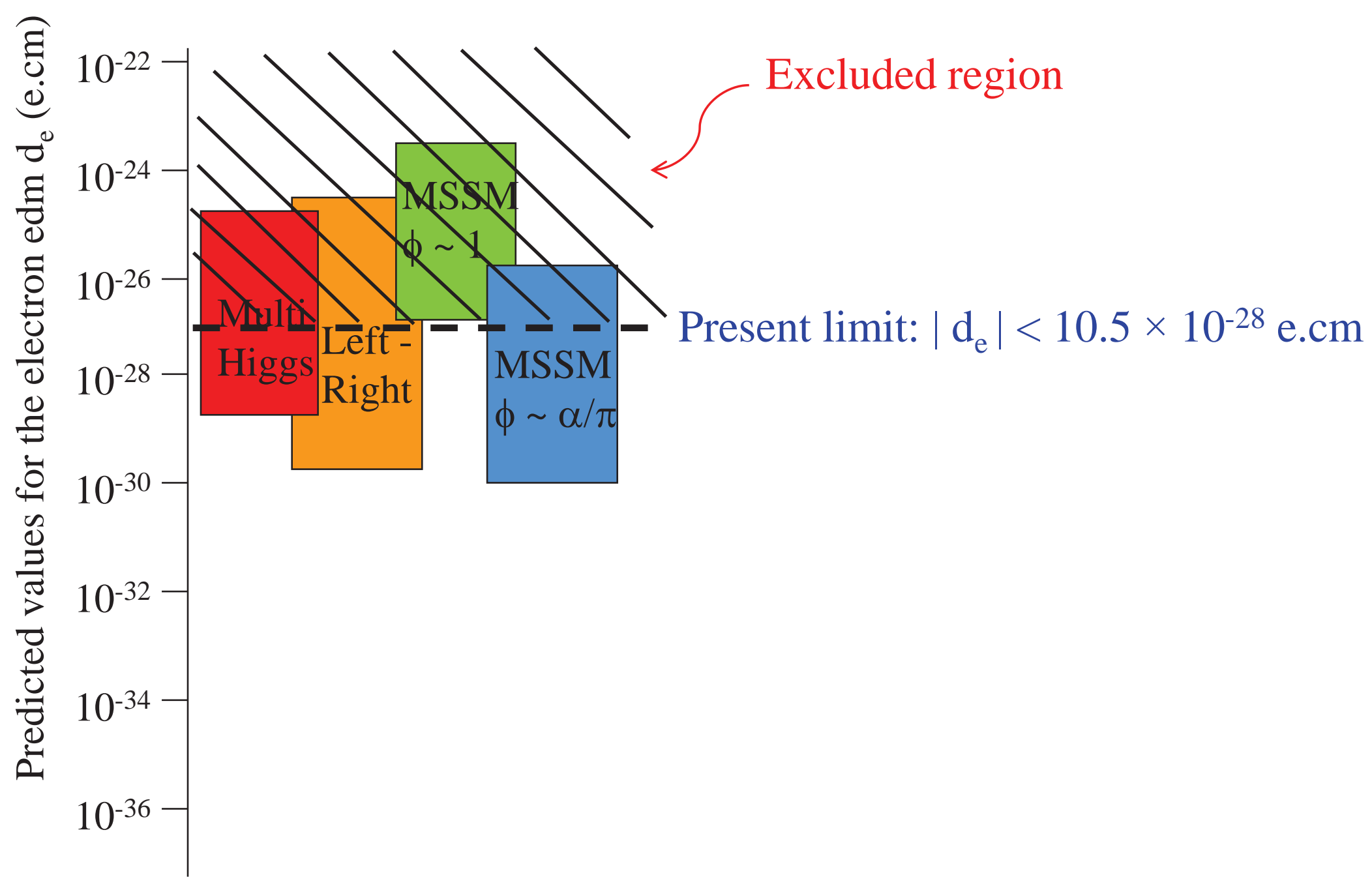

Standard Model 


\section{Consequences of neutron EDM measurements}

For the neutron there is a CP-violating term in the QCD Lagrangian, parametrized by $\theta$.

This gives a neutron EDM: $\quad d_{n} \sim \theta\left(6 \times 10^{-17}\right)$ e.cm

So the experimental upper limit for the neutron EDM gives $|\theta|<5 \times 10^{-10}$

This fine tuning of the $\theta$ parameter is the strong $C P$ problem

This problem can be resolved by introducing the axion

If we assume that (for whatever reason) the $\theta$ contribution is suppressed to zero, then $d_{n}$ in the Standard Model is smaller than $10^{-32}$ e.cm 


\section{Constraints from EDM measurements}

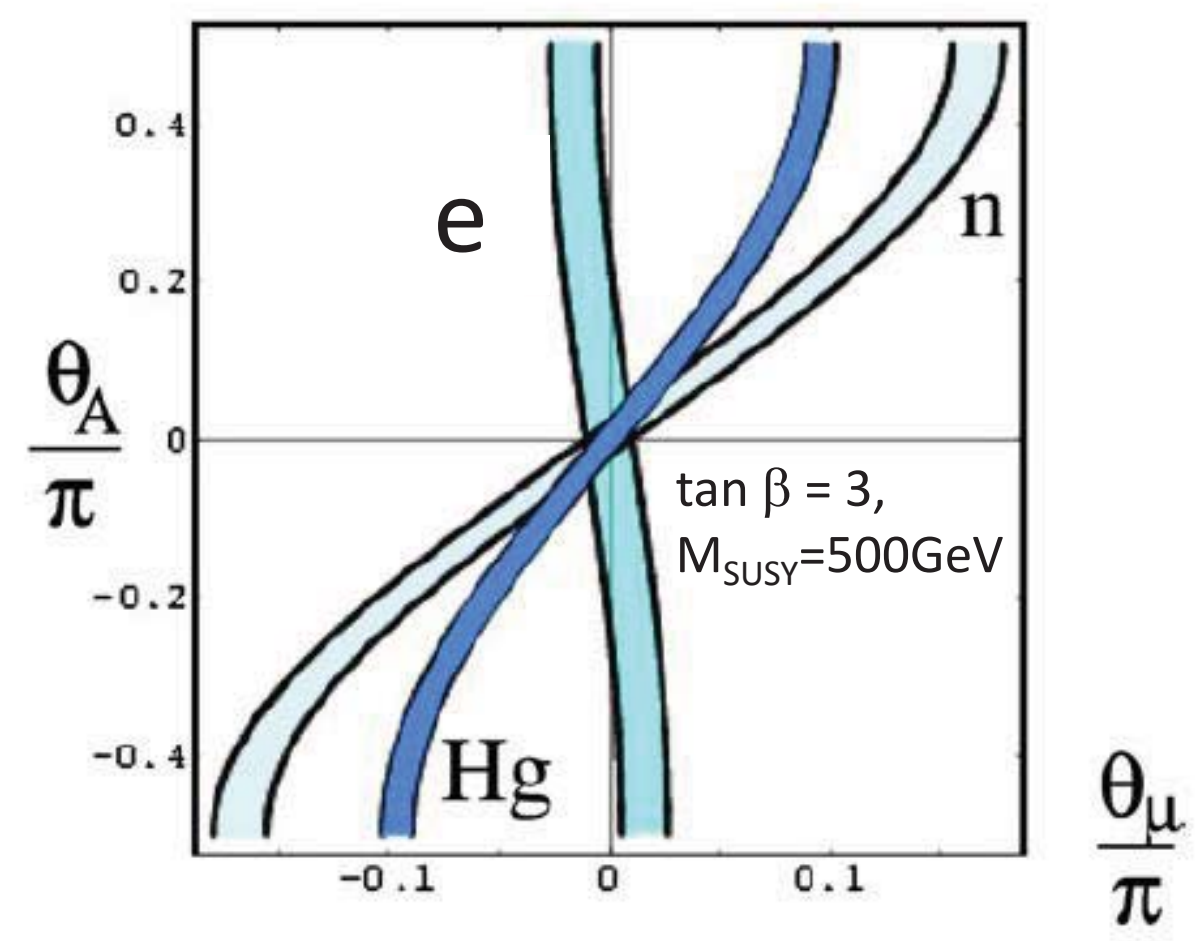

M. Pospelov, A. Ritz, Annals of Physics 318, 119 (2005) 


\section{Acknowledgements}

Thanks to Jony Hudson, Ben Sauer and Ed Hinds for their help and advice. 